\title{
3D Photonics as Enabling Technology for Deep 3D DRAM Stacking
}

\author{
Sebastian Werner, Pouya Fotouhi, Xian Xiao, \\ Marjan Fariborz, S.J. Ben Yoo \\ University of California, Davis, CA \\ \{swerner,pfotouhi,xxxiao,mfariborz,sbyoo\}@ucdavis.edu
}

\begin{abstract}
3D stacking improves bandwidth, energy, and latency of DRAMs by exploiting shorter and more abundant wiring in three dimensions. While future stacks are predicted to provide tens of DRAM layers, TSV pitches are bound to stop decreasing due to physical limitations, requiring either large area overheads or energy for higher pin data rates to preserve bandwidth scaling. In addition, deep 3D DRAM stacks increase the average number of TSVs hops to reach the appropriate DRAM layer. Recent advances in vertical silicon-photonic interconnects now allow diameters of $1-2 \mu \mathrm{m}$ which results in high bandwidth density for optics. In this paper, we explore the benefits and architectural implications of vertical optical interconnects in terms of area, power, and performance. We propose a hierarchical approach to stacking 3D DRAM to tens of layers by utilizing sub-stacks which are optically-interconnected to a memory interface on the processor die. Our results show that photonics could be a key enabler for deep-3D DRAM offering at least $2 \times$ interconnect area savings compared to TSVs for the same bandwidth with comparable performance and less power.
\end{abstract}

\section{KEYWORDS}

3D DRAM, silicon photonics, die stacking

\section{INTRODUCTION}

Era of 3D Integration. The slowing down of Moore's law and Dennard scaling has led to research on 'more-than-Moore' technologies [125] to preserve compute and energy improvements in future systems. 3D integrated circuits (3D ICs) [71-73] is one such technology offering several key benefits from vertically integrating and interconnecting silicon dies in the same package such as (i) reduced horizontal wire lengths-compared to 2D ICs for a fixed amount of gates-lowering latency and energy on the interconnect, (ii) higher memory bandwidth by relieving processor-to-memory interconnects from off-chip pin count constraints, and (iii) tight integration of heterogeneous, possibly incompatible processor and memory technologies [119].

Permission to make digital or hard copies of all or part of this work for personal or classroom use is granted without fee provided that copies are not made or distributed for profit or commercial advantage and that copies bear this notice and the full citation on the first page. Copyrights for components of this work owned by others than ACM must be honored. Abstracting with credit is permitted. To copy otherwise, or republish, to post on servers or to redistribute to lists, requires prior specific permission and/or a fee. Request permissions from permissions@acm.org.

MEMSYS '19, September 30-October 3, 2019, Washington, DC, USA

(c) 2019 Association for Computing Machinery.

ACM ISBN 978-1-4503-7206-0/19/09 . \$ $\$ 15.00$

https://doi.org/10.1145/3357526.3357559

\author{
George Michelogiannakis, Dilip Vasudevan \\ Lawrence Berkeley National Laboratory, Berkeley, CA \\ \{mihelog,dilipv\}@lbl.gov
}

In particular, die stacking which processes each layer separately prior to vertically stacking them together has emerged as a costefficient technique in industry as it reduces manufacturing time by allowing the processing of dies in parallel and allows use of conventional fabrication processes. 3D-stacked DRAM (e.g., HBM [49], HMC [79] and Wide IO [126]) are the most notable technologies that use this technique by stacking DRAM dies. This exploits abundant vertical interconnects and shorter wire lengths to improve memory bandwidth and access latency-two major reasons for the 'memory wall' [88] and memory bandwidth constraints of modern and future workloads.

Future Outlook of 3D ICs. Looking ahead, given the growing working sets and memory access requirements of various key application domains (e.g., HPC, big data, ML) coupled with the exorbitant cost of large SRAM caches and shrinking feature sizes below $7 \mathrm{~nm}$, future systems will likely increasingly rely on 3D-stacked DRAM to satisfy memory demands. In fact, the ITRS 2.0 roadmap projects improvements in waver-thinning and integration technology to shrink die thickness from currently $50-100 \mu \mathrm{m}$ to $5-15 \mu \mathrm{m}$ in the next 15 years [48], allowing deep-3D ICs with tens of stacked layers within a fixed-height stack and in turn large in-package memory capacity improvements [103] $]^{1}$. Memory bandwidth, however, depends on the interconnect density of vertical interconnects, which would have to scale proportionally to the number of DRAM layers to maintain per-layer bandwidth without excessive area overheads on the processor and memory dies. Unfortunately, Through-silicon Vias (TSVs) and Microbumps ( $\mu$ Bumps), which are the state-of-theart technologies, are not expected to scale down appreciably in size.

Vertical Interconnect Density Challenges. Pitch sizes of TSVs and microbumps limit vertical interconnect density and depend on their diameter and spacing between components, which will face key challenges in the future:

(i) Wafer thickness limits. TSV diameters are limited by the TSV depth (vertical length through the die) as the TSV aspect ratio (depth to diameter) must be within certain limits for reliability and manufacturability (currently 10:1-20:1 [31, 46, 101]). Consequently, die thickness determines TSV depth and in turn TSV diameter, making wafer thinning technologies key for reducing TSV pitch. However, wafer thinning increases cost through additional fabrication steps and forces thinner wafers to be smaller in size to avoid wafer cracking, lowering manufacturing volume $[20,29,58]$. 6-10 $\mu$ m have been reported as practical limits of silicon layer thickness of stacked dies in the future $[1,2]$.

\footnotetext{
${ }^{1}$ The most recent HBM 2.0 generation allows up to 12 layers of stacked DRAM with $1 \mathrm{~GB}$ capacity per layer $[50,77]$
} 
(ii) Bonding alignment precision. TSVs smaller in diameter require high-precision bonding alignment technologies to create contacts between TSVs of stacked dies. This process is projected to be limited to $0.5-1 \mu \mathrm{m}$ in the future $[1,2]$. Microbumps (typically solder) between layers are used to mitigate misalignment of TSVs and are thus larger in diameter than TSVs. Scaling microbumps $<10 \mu \mathrm{m}$ is prone to defects due to higher risk that microbumps will be slid during bonding. Recently proposed "bumpless" solutions allow practical scaling down to $5 \mu \mathrm{m}$ by encasing bumps with polymers, allowing them to keep up with TSV pitch scaling. However, for current and likely future technologies, microbump pitches are larger than TSV pitches, therefore further reducing interconnect density.

(iii) Keep out zones. TSV fabrication causes thermo-mechanical stress around TSVs which renders adjacent silicon circuitry defective and thus requires keep-out zones around TSVs, increasing TSV pitch even further [43, 99, 114, 122]. Recent analytical studies explored that the induced thermo-mechanical stress between TSVs becomes insignificant if TSV pitch is thrice its diameter [7], further limiting TSV interconnect density.

Monolithic 3D integration has been explored as a solution to the TSV limitations of die stacking and involves a sequential device process in which the front-end processing (building the device layer) is repeated on a single wafer to construct multiple active device layers prior to inserting interconnects among the devices in the back-end process. Although monolithic 3D-ICs offer throughsilicon connections with $<50 \mathrm{~nm}$ diameter $(10.000 \times$ higher areal density than TSVs $[1,2])$, mass production will require a practical solution to several key challenges such as lack of EDA design tools, aligning circuits with transistor-level accuracy, and reducing large cost overheads of layering heterogeneous memory and logic circuitry in a single device that requires a change of conventional fabrication processes [21, 78, 92].

In addition, both TSVs and monolithic 3D integration inevitably impose energy per communication that is proportional to the number of DRAM layers that have to be traversed to reach the desired layer, since all intermediate layers have to be traversed. For the same reason and assuming constant area per layer, bandwidth that escapes a logic layer is shared among traffic that reach all DRAM layers assigned to that logic layer (potentially tens or hundreds). Therefore, the bandwidth per DRAM layer that any one in particular logic layer has available is inversely proportional to the number of DRAM layers.

Silicon Photonics as an Enabling Technology. Silicon photonics (SiPhs) are a promising "more-than-Moore" technology that allows integrating optical links compatible with CMOS circuitry into processors or memories. Optical communication provides high interconnect density through wavelength-division multiplexing (WDM) which allows data to be transmitted on tens of wavelengths in parallel on the same link, next to speed-of-light signaling that is practically distance-independent in terms of energy consumption. Nevertheless, vertical optical interconnection for 3D-stacked systems has, so far, not been explored because previously demonstrated optical TSVs exhibited $\sim 100 \mu$ m diameters [95]-too big to compete with current TSV technologies despite the bandwidth density benefits of WDM. However, recently demonstrated vertical optical vias significantly change this playing field by featuring
$1-2 \mu \mathrm{m}$ pitches [128]-possibly making them a key enabling technology for high-density 3D optically-interconnected DRAM stacks. More importantly, these vias can be fabricated with standard CMOS processes, allow alignment errors within tens of nanometer, do not require additional packaging steps (common in SiPhs) [128], and can be combined with all the cost benefits of die stacking.

In this paper, we explore the benefits of vertical SiPh interconnects for future deep-3D ICs and architectures that follow from it. We study how deep 3D stacks vertical SiPh interconnects enable compared to monolithic 3D integration and TSVs. In particular, we make the following contributions:

- We conduct a detailed analysis of current and future DRAM 3D integration scaling trends using monolithic 3D integration and TSVs. We outline hard challenges in terms of interconnect area overheads and bandwidth density. We identify that this will limit scaling the number of DRAM layers in future memory generations and consequently limit storage density and DRAM access energy.

- Based on our observations about electrical vertical interconnects, we analyze recently developed $\mu \mathrm{m}$-scale $\mathrm{SiPh}$ vertical interconnects as a means to overcome the area and bandwidth limitations of TSVs. We show that their attributes can provide deep 3D DRAM stacks with tens of layers, scaling the memory channel bandwidth of current HBM technology to tens of DRAM layers with low interconnect area overheads and no modifications to DRAM process technology.

- We propose a 3D DRAM architecture based on vertical SiPh interconnects that scales DRAM stacks to tens of DRAM layers by stacking 'sub-stacks' on top of each other, where each sub-stack consists of multiple DRAM layers and a SiPh interface layer that is optically-connected to a DRAM interface layer that, in turn, is placed on top of the connected processor. Our simulation results show that our design, despite requiring electrical-to-optical conversion, exhibits negligible performance overhead and slightly reduces power consumption over a range of HPC workloads compared to conventional TSV based DRAM stacks, while halving interconnect area requirements for the same layer-to-layer bandwidth.

\section{BACKGROUND AND MOTIVATION}

\subsection{D Stacked DRAM: State-of-the-art}

Figure 1 depicts the layout of a die layer in an HBM 2.0 stack with four DRAM layers (4Hi) on top of a logic layer with one memory controller per channel one per channel. Each layer contains two physical memory channels $(\mathrm{CH})-8$ channels for the entire stackthat are operated completely independently of each other by different memory controllers for high memory-level parallelism (MLP)needed to keep bus utilization high in the face of high DRAM access latencies. HBM 2.0 further divides $\mathrm{CHs}$ into two pseudo-channels (PS-CHs) which share the CH's address/control (ADD/CMD) bus but have their own 64-bit wide IO interface which further helps to increase MLP $[65,90]$. Each CH therefore has a 128-bit interface, leading to an $8 \times 128=1024-$ bit interface between the processor/logic layer and the HBM stack. Pin data rates of up to $2.4 \mathrm{~Gb} / \mathrm{s}$ provide up to $307 \mathrm{~GB} / \mathrm{s}$ memory bandwidth. Typical dimensions of a 4Hi HBM stack including logic layer with memory controllers are 


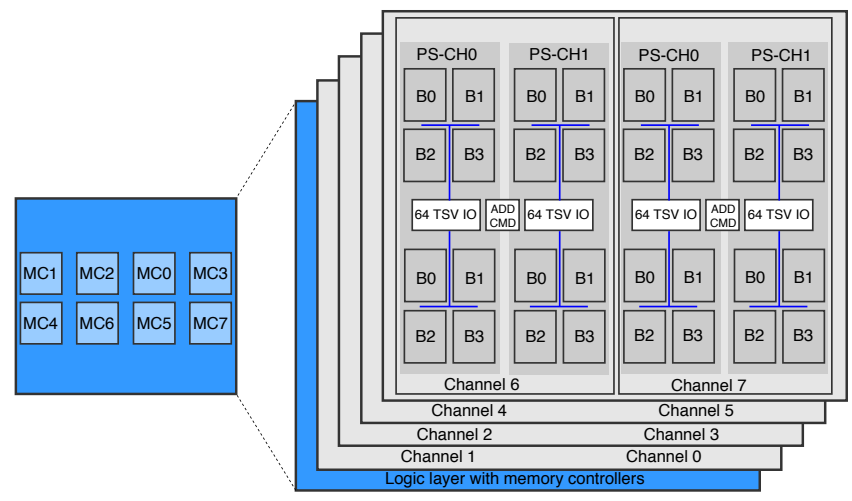

Figure 1: HBM 2.0 stack [49] with four layers, each with two physical channels $(\mathrm{CH})$, atop a logic layer with one memory controller (MC) per channel. Each $\mathrm{CH}$ consists of two pseudo-channels (PS-CHs) with 8 DRAM banks (B) sharing a 64-bit wide bus (address/command bus is shared between two PS-CHs).
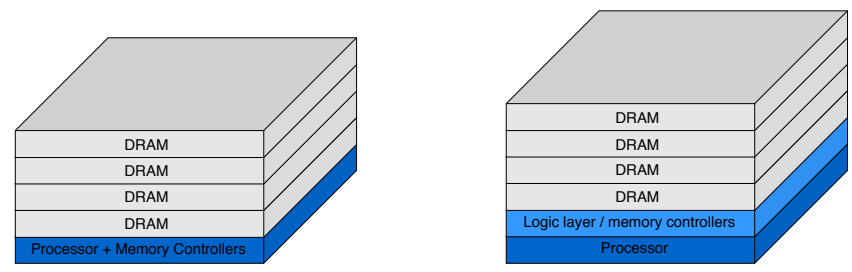

Figure 2: 3D-stacked chip integration options: separate logic layer with memory controllers vs. memory controllers integrated into processor chip.

$5.5 \mathrm{~mm} \times 7.7 \mathrm{~mm} \times 0.48 \mathrm{~mm}$ [68] (i.e., $\sim 100 \mu \mathrm{m}$ thick dies). System integration in pure-3D systems of HBMs can either stack them atop a separate logic layer containing memory controllers (one per channel) or directly atop the processor containing memory controllers (Figure 2).

Figure 3a illustrates the side view of a DRAM stack atop of a processor die. In this example, DRAM layers are connected through TSVs, which provide vertical connectivity between the layers, and microbumps, which are the contacts between dies necessary to mitigate misalignment issues. As shown in Figure 3b, TSVs vertically connect stacked silicon dies by passing completely through and connecting to the metal layers within the dies. To achieve that, TSV cavities are etched into silicon dies from the top which are subsequently thinned from the bottom to expose the TSV. To reduce development cost through high-volume manufacturing, the stacked DRAM layers are typically uniform in design. Therefore, TSV cavities for all 1024 TSVs (in HBM 2.0) are etched into each die layer, but the DRAM banks on the dies only connect to the 128 TSVs corresponding to their physical channel. Non-uniform designs of DRAM layers avoids unnecessary TSV area overheads, but significantly increase development and manufacturing cost [59].

Figure 3a illustrates that the bandwidth that escapes the logic layer is a fundamental limiting factor to the bandwidth per DRAM

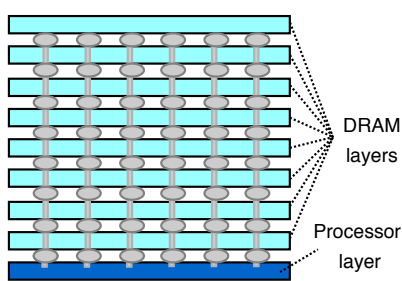

(a) Side view of a 3D DRAM stack atop a processor.

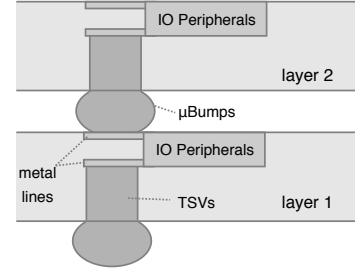

(b) Detailed view of two layers interconnected with TSVs and microbumps.
Figure 3: Example 3D integrated system [59] with processor and DRAM stack vertically-interconnected with TSVs and microbumps. IO peripheral circuitry within device layers are the interface between vertical links and $2 \mathrm{D}$ circuitry.

layer. That is because all DRAM traffic escaping the logic layer has to use the TSVs that connect to that logic layer, regardless of destination. Therefore, the bandwidth per DRAM layer in this example is-assuming an even distribution-the total bandwidth escaping the logic layer divided by the number of DRAM layers. In addition, it is apparent that placement of data to DRAM layers is important because data placement affects the number of TSVs any particular request has to traverse and thus the required energy and possibly contention with other requests.

\subsection{D Stacked DRAM: Outlook}

Future systems and applications will drive the demand for improved memory capacity, bandwidth, and access latency. 3D-stacked DRAM has played a critical role in accelerating computation in various modern workloads which have to process large data sets that are expected to further increase in years to come [88]. In addition, larger numbers of (heterogeneous) processors will increase computing power, putting further strain on memory. Consequently, memory bandwidth, latency, and capacity are expected to remain first-order metrics for future 3D-stacked DRAMs.

Scaling memory capacity of a DRAM stack of fixed horizontal dimensions ultimately depends on the total number of layers, which is limited by (i) thermals dictating the height of the stack and (ii) manufacturing technology (i.e., testing and integration) [103]. Forecasts by the ITRS 2.0 roadmap predict current die thickness $(50-100 \mu \mathrm{m})$ to shrink to $5-15 \mu \mathrm{m}$ in the next 15 years, a projection generally supported by trends in commercially-available stacked DRAM designs, e.g. layers in HBM offers increased from originally 4 layers to now $12[50,77]$. In parallel, rapid advances in thermal management and cooling oin $3 \mathrm{D}[56,97,127]$ provide promising outlooks for the viability of deep 3D stacks, especially for DRAM stacks due to their lower average activity factor.

Such improvements will pave a viable path towards tens of stacked layers within a fixed-height stack in years to come, providing deep 3D DRAM stacks. For instance, for $10 \mu \mathrm{m}$ thick dies, more than 40 layers could fit into the $0.48 \mathrm{~mm}^{2}$ high stack of the $4 \mathrm{Hi}$ HBM [68]. This poses the question how DRAM stacks with such a higher number of layers should be designed and what their impact on memory latency and bandwidth is. 


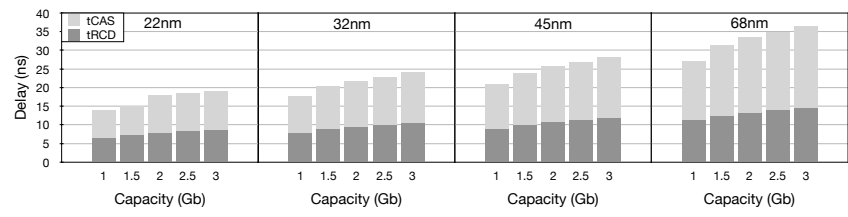

Figure 4: DRAM access latency for different chip capacities.

\subsection{Memory Latency in Future Deep-3D Stacked DRAM}

Looking forward, exploiting thinner dies to scale capacity by adding DRAM layers, rather than increasing die sizes horizontally, offers lower memory latency and energy due to overall shorter interconnect lengths. Consequently, we project future designs to aim for scaling memory capacity by adding DRAM layers to attain low memory access latency, which is known to have a large impact on computing system performance [28].

Latency reductions have notoriously been stagnating due to bus, precharge, row and column access latency $\left(t_{R A S}\right)$ caused by relatively long planar interconnects and slow transistors of the DRAM process $^{2}-$ a well-understood reason for the memory wall [103]. As electrical interconnect latency and energy scale poorly compared to transistors for shrinking technology nodes, only reducing wire length will provide lower access latency without energy overheads. As an example, Figure 4 depicts the impact of increasing capacity of a DRAM chip/die on memory access time (tCAS + tRCD) for different technology nodes in a commodity DRAM technology process (modeled with Cacti [87]). At 22nm, doubling capacity from $1 \mathrm{~Gb}$ to $2 \mathrm{~Gb}$ causes a $30 \%$ increase in access latency, clearly outlining the latency overheads of increasing memory capacity $2 \mathrm{D}$.

Although a number of papers previously proposed architectural modifications of DRAM internals reducing wire lengths (e.g., reducing the number of cells per bitline/wordline within a bank/subarray to lower wire capacitance for increased drive speed) that, in turn, successfully decrease DRAM access latency [5, 6, 22-24, 54, 59-63, $102,107]$, these often incurr area, costs (for more sense-amplifiers) and interconnect (more banks accessing the bus) overheads, limiting their potential to fundamentally break the latency issue of the memory wall.

Consequently, there is a strong drive towards maintaining DRAM die sizes as much as possible to avoid increases in memory latency when scaling memory capacity, which can be provided by increasing the number of DRAM layers in the future. However, while exhibiting the best capacity scaling approach from a latency perspective, maintaining memory bandwidth per layer will require TSV pitches to decrease proportionally to provide bandwidth scaling without prohibitive area overheads, which is doubtful as we will see in the following.

\footnotetext{
${ }^{2}$ High performance transistors as in eDRAM can bring DRAM latency closer to SRAM at the cost of much shorter refresh cycles due to the lower threshold voltages, which is impractical for high-capacity systems.
}

Table 1: Vertical interconnect technology comparison of pitch sizes

\begin{tabular}{ccc}
\hline & Commercially-available & Research demonstrations \\
\hline TSV & $15-20 \mu \mathrm{m}[15,30,74,100]$ & $5-10 \mu \mathrm{m}[15,25,41,44,100,112,113]$ \\
Microbump & $30-55 \mu \mathrm{m}[35,75,80,129]$ & $6-20 \mu \mathrm{m}[12,15,118]$ \\
Bumpless TSV & - & $5-10 \mu \mathrm{m}[32,33,91]$ \\
\hline MIV & - & $50 \mathrm{~nm}[17,104,120]$ \\
\hline
\end{tabular}

Table 2: Number of TSVs required to maintain bandwidth to each die in HBM2.0 for increasing number of layers.

\begin{tabular}{lccccccccc}
\hline \#Layers & 8 & 12 & 16 & 20 & 24 & 28 & 32 & 36 & 40 \\
\hline \#TSVs & 2048 & 3072 & 4096 & 5120 & 6144 & 7168 & 8192 & 9216 & 10240 \\
\hline
\end{tabular}

\subsection{Memory bandwidth in future deep-3D Stacked DRAM}

Interconnect density is a critical metric for determining memory bandwidth in 3D-stacked DRAMs as it determines the number of links, and thus maximum attainable bandwidth, within a certain area. Microbumps are the inter-layer contact and typically larger in pitch than TSVs and, as a result, determine the maximum attainable inter-layer interconnect density. Nevertheless, TSV diameters, pitches, and keep-out zones significantly impact silicon die area as they are etched through the entire silicon die. Improvements in integration and manufacturing technology has lead to shrinking of diameters and pitches of both TSVs and microbumps in recent years. Table 1 lists the pitch sizes of commercially-available as well as state-of-the-art research demonstrations of TSVs, microbumps, and bumpless technologies.

Unfortunately, as discussed in Section 1, scaling down TSV and microbump pitches and diameters will soon stop due to limitations in bonding alignment technology $(0.5-1 \mu \mathrm{m})$, stacked silicon die thickness $(6-10 \mu)[1,2]$, and associated TSV aspect ratios. These factors will stop TSV diameter scaling around $1 \mu \mathrm{m}$ and TSV pitches around $2-3 \mu \mathrm{m}^{3}$.

2.4.1 Impact of TSV pitch on area. Dimensions of TSV pitches become more meaningful when related to the bandwidth demands of 3D-stacked DRAMs, particularly for increasing number of layers. Table 2 lists the number of TSVs that would be required to maintain the per-layer bandwidth of an HBM 2.0 stack (with fixed-sized dies of HBM 2.0) when scaling HBM 2.0 to higher numbers of layers based on two physical channels per layer requiring $2 \times 128-$ bit $=$ 256 TSVs (assuming the same pin data rate). To no surprise, this leads to thousands of TSVs between the processor and DRAM stack in the future.

For such high quantities of TSVs, TSV pitch size has a significant impact on the overall area occupied by TSVs. Figure 6 shows the area requirements for increasing number of layers on each die (Figure 5a) and of the entire stack, i.e. of all layers combined (Figure 5b). Pitch sizes represent commercially-available TSVs $(20 \mu \mathrm{m})$, state-of-theart research demonstrations $(10 \mu \mathrm{m} \$ 5 \mu \mathrm{m})$, as well as an optimistic

${ }^{3}$ An et al. [7] found that thermo-optical stresses are insignificant for pitch $=3 \times$
diameter, so $2 \times 1 \mu \mathrm{m}$ pitch is an optimistic, while $3 \times 1 \mu \mathrm{m}$ a conservative estimate. 


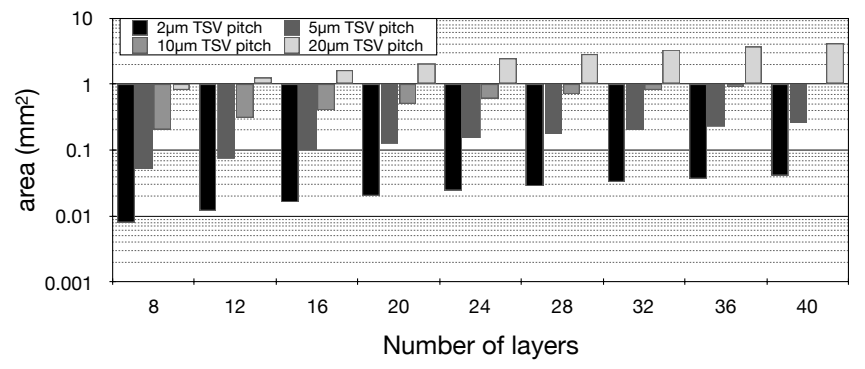

(a) Total TSV area on each die layer.

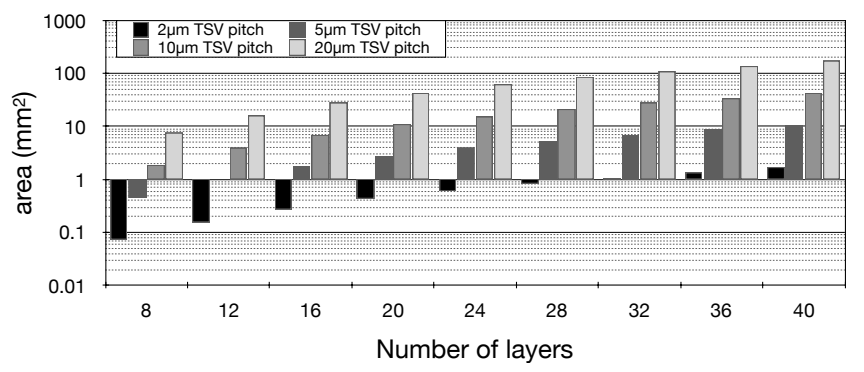

(b) Total TSV area of the whole stack.

Figure 5: TSV area requirements of DRAM stacks with different number of die layers. Values are based on the HBM2.0 standard which provides two channels per die with a 128-bit wide interface [49]. Dies are assumed to be fabricated uniformly for low-cost high-volume manufacturing, i.e. each die has all TSVs etched into it [59].

projection of TSVs that might be obtained in the future $(2 \mu \mathrm{m})$. We observe that for currently commercially-available TSV pitches of $20 \mu \mathrm{m}$, future stacks in the short term (20 layers) and the long term (40 layers) will have to dedicate TSV area of $2 \mathrm{~mm}^{2}$ and $4 \mathrm{~mm}^{2}$ per DRAM die, and $40 \mathrm{~mm}^{2}$ and $160 \mathrm{~mm}^{2}$ for the entire stack.

Generally, aggressive future projections of $2 \mu \mathrm{m}$ will be necessary to avoid exorbitant area overheads which could otherwise be used for memory cells in the DRAM layer. Figure $6 \mathrm{a} / 6 \mathrm{~b}$ puts the TSV area overhead in relation to the number of memory cells that could fit into the same area space for a memory density of $0.2 \mathrm{~Gb} / \mathrm{mm}^{2}$ that corresponds to a 30nm Micron technology [27]. We observe that TSV pitches $>10$ lead to area overheads that could be used for several (or even tens) of Gbs of capacity. Even $5 \mu \mathrm{m}$ pitches lead to Gbs of wasted capacity. In fact, a further scaling of DRAM to smaller technology nodes (e.g., $22 \mathrm{~nm}, 14 \mathrm{~nm}$ ) could increase this wasting of memory capacity significantly. Note that, these TSV area overheads also potentially lead to longer wires between the processor and the DRAM banks, causing higher memory access energy and latency.

2.4.2 Impact of Pin data rates. A straight-forward way to reduce large TSV area overheads while maintaining bandwidth is scaling bandwidth by increasing pin data rates, which can be much higher than $2.4 \mathrm{~Gb} / \mathrm{s}$ in HBM 2.0 (for instance, GDDR6 delivers up to $16 \mathrm{~Gb} / \mathrm{s} /$ pin [111]). Data rates in HBMs, despite increasing from $1 \mathrm{~Gb} / \mathrm{s}$ (first HBM generation [77]) to $2.4 \mathrm{~Gb} / \mathrm{s}$ (recently announced [50]), have traditionally been relatively low as the wide

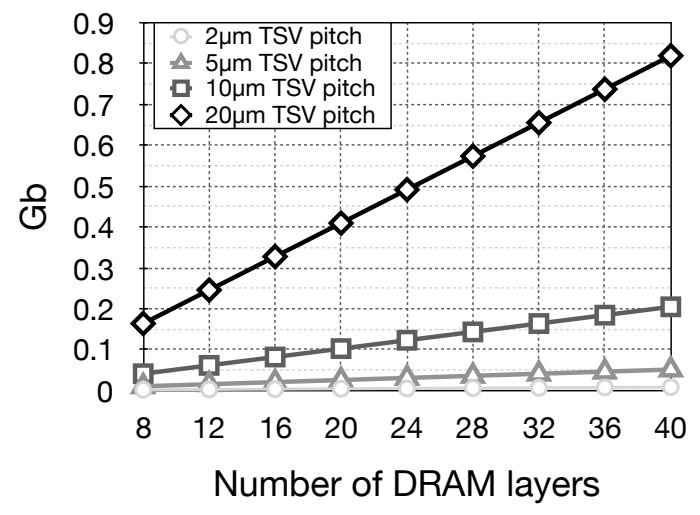

(a) Gb of DRAM cells fitting into TSV area per layer.

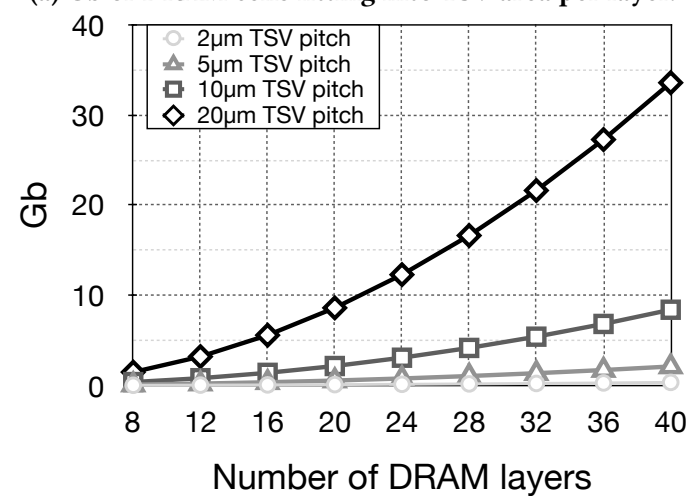

(b) Gb of DRAM cells fitting into TSV area for entire stack.

Figure 6: Relationship between TSV area overheads and memory capacity that could be used within the same dimensions based on $0.2 \mathrm{~Gb} / \mathrm{mm}^{2}$ of a 30nm Micron memory chip [27].

IO interface enabled by abundant TSV resources offers sufficient bandwidth (this is in contrast to conventional 2D DRAMs which rely on high-speed narrow IO interfaces due to limit off-chip pin availability).

In fact, low-data-rate pins are a major benefit of HBMs as they allow the DRAM IO circuitry to operate well within DRAM transistor process capabilities (clock frequency within DRAM chips has settled at $200 \mathrm{MHz}$ [111]), which are much slower than the CMOS circuitry in the memory interface. Therefore, increasing pin data rates would require SERializer/DESerializer (SERDES) circuitry, including phase-locked-loop (PLL) and buffering resources, at the memory controller and within the DRAM layers to up-/down-convert data rates, as well as sufficiently large prefetch buffers to actually take advantage of the available bandwidth. Higher data rates, the corresponding higher clock frequencies, and the circuitry overheads not only lead to significant increases in power consumption, but also induce area overheads [93], thereby completely eliminating any area savings gained by opting for fewer but higher clock TSVs and therefore not offering an effective solution to the TSV area overhead problem. 
2.4.3 Number of memory controllers. Maintaining two physical channels per layer and one memory controller per channel leads to a large number of memory controllers for deep-3D DRAM stacks. Coupled with the slowing down of Moore's law and fixed 2D die sizes to avoid memory access latency overheads, this inevitably leads to area and layout concerns on the logic die underneath the DRAM stack. In particular, this raises the question of how many DRAM layers (and thus, channels) can be deployed for fixed 2D die sizes before the memory controllers become the bottleneck.

To answer this question, we model a memory controller in McPat [69] for a 22nm technology node with a configuration similar to a memory controller for a physical channel in an HBM2.0, with $2 \mathrm{~Gb} / \mathrm{s}$ pin data rates, a 128 -bit wide DDR interface, and a 64 entry wide request buffer. For this configuration, McPat reports an area footprint of $1.38 \mathrm{~mm}^{2}$ (including front end engine, transaction engine, and PHY [69]). For current HBM dimensions of $5.5 \mathrm{~mm} \times 7.7 \mathrm{~mm}=42.35 \mathrm{~mm}^{2}$, this would mean that $\sim 30$ memory controllers could fit underneath the DRAM stack-corresponding to 15 layers with 2 memory channels each (spacing and layout considerations would decrease the number of layer further).

Although memory controller sizes can vary based on their complexity (e.g., QoS support) and optimization target (low-power vs. high-performance), these do not fundamentally change this issue, especially since much of the area is taken up by the analog circuitry in the PHY to support the bandwidth (i.e., width and pin data rate) of the HBM interface. Deep-3D DRAM stacks will therefore have to solve this issue to avoid latency overheads of horizontally increasing die sizes.

\subsection{Memory Latency and Bandwidth in Modern Workloads}

Modern workloads exhibit increasing memory capacity and bandwidth demands. The task of accommodating these workloads with low access latency has led to the implementation of expensive SRAM caches that can occupy up to $40 \%$ of a processor's die area [105]. 3D-stacked DRAM is typically deployed as another level in the cache hierarchy (rather than main memory) $[52,66,81]$, re-architected and optimized for low access latency [103]. Simulation results of a recent study [103] indicate that the relatively-larger capacity of stacked DRAM caches outperforms SRAM caches despite higher access latency for server workloads as they can accommodate more of the large working sets [103].

We take this study further and investigate the impact of a range of different memory bandwidth and latency data points to quantify their significance to a range of modern HPC workloads to project impact on future stacked DRAMs. We use gem5 [16] with garnet [3] in full-system mode to simulate a range of HPC workloads from the NAS Parallel Benchmark suite [11], Splash-2x/Parsec3.0 [124], and Rodinia [26]. Table 3 lists the system configuration of the considered designs, a sketch of which is also shown in Figure 7. We assume a 16-core system in which 4 cores clustered per router in a $2 \times 2$ Mesh NoC share an L2 slice. SRAM LLC depicts a typical current baseline design with a large L2 SRAM caches with 8 cycles access latency estimated with CACTI [87]. In addition, we compare this to DRAM LLC which has low L2 SRAM capacity, thus more relying on stacked DRAM (L3). We assume one HBM 2.0 module per router

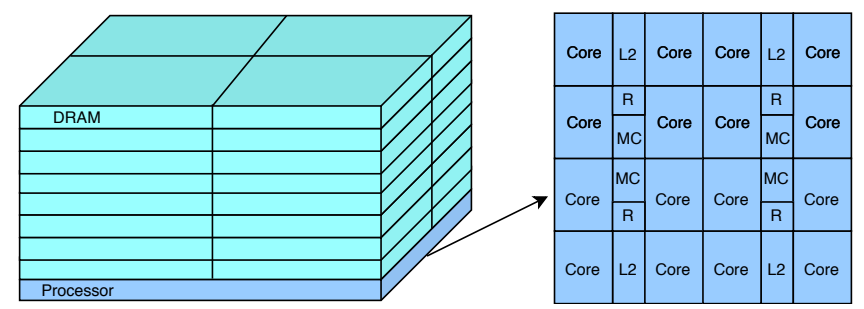

Figure 7: System configuration illustration. Each router is connected to four cores, an L2 cache slice shared by these four cores, and a memory controller for DRAM access, as well as to routers through a $2 \mathrm{D}$ mesh.

Table 3: System configuration details.

\begin{tabular}{cccc}
\hline & L2 size & $\begin{array}{c}\text { Memory } \\
\text { bandwidth }\end{array}$ & tRP-tRCD-tCL-tRAS \\
\hline SRAM LLC & $4 \times 16 \mathrm{MB}$ & $256 \mathrm{~GB} / \mathrm{s}$ & $15-15-15-33 \mathrm{~ns}$ \\
DRAM LLC & $4 \times 128 \mathrm{kB}$ & $256 \mathrm{~GB} / \mathrm{s}$ & $15-15-15-33 \mathrm{~ns}$ \\
DRAM LLC BW 22 & $4 \times 128 \mathrm{kB}$ & $512 \mathrm{~GB} / \mathrm{s}$ & $15-15-15-33 \mathrm{~ns}$ \\
DRAM LLC BW $\times 4$ & $4 \times 128 \mathrm{kB}$ & $1024 \mathrm{~GB} / \mathrm{s}$ & $15-15-15-33 \mathrm{~ns}$ \\
DRAM LLC Lat1 & $4 \times 128 \mathrm{kB}$ & $256 \mathrm{~GB} / \mathrm{s}$ & $12-12-12-28 \mathrm{~ns}$ \\
DRAM LLC Lat2 & $4 \times 128 \mathrm{kB}$ & $256 \mathrm{~GB} / \mathrm{s}$ & $10-10-10-24 \mathrm{~ns}$ \\
\hline & $16 \times 86 \mathrm{O} 3$ cores @ 2GHz, L1I/D 32kB 8-way, \\
General setup & $2 \mathrm{D} \mathrm{2 \times 2} \mathrm{Mesh} \mathrm{NoC} \mathrm{with} \mathrm{Clustering} \mathrm{of} \mathrm{4,}$ \\
& \multicolumn{4}{c}{4 x HBM2.0 (one per router) } \\
\hline
\end{tabular}

(four in total) in all designs. In addition to the baseline DRAM LLC which is configured according to the JEDEC specifications of the HBM 2.0 interface [49], we vary the memory bandwidth (BWx2 and BWx4) and latency (Lat1 and Lat2) to values potentially attained in future generations.

Figure $8 \mathrm{a}, 8 \mathrm{~b}$, and $8 \mathrm{c}$ show the total application execution time, average memory access latency to the 3D-stacked DRAM, and the average queuing latency at the memory controllers, respectively.

\subsection{Summary}

In summary, we conclude that memory access latency remains critical for system performance, to which delay on planar horizontal interconnects from/to banks on the DRAM dies contribute significantly. Consequently, exploiting higher stacks to scale capacity while maintaining 2D DRAM die sizes appears the superior design choice going forward. However, enabling such deep-3D system scaling introduces several key challenges that must be overcome first:

- Memory bandwidth continues to be critical for performance and to extract memory-level parallelism. However, proportional scaling of memory bandwidth with the number of DRAM layers in a stack will lead to large area overheads for TSV area if TSV pitches are not $<5 \mu \mathrm{m}$. This not only wastes area that could otherwise be used for logic gates or DRAM cells, but also increase horizontal wire lengths as banks must be placed further apart and complicates physical layout. 


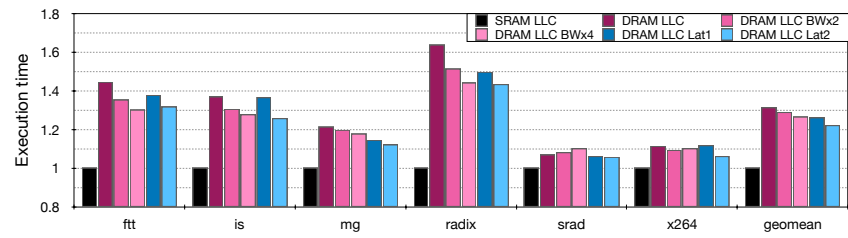

(a) Total application execution time normalized to SRAM L2.

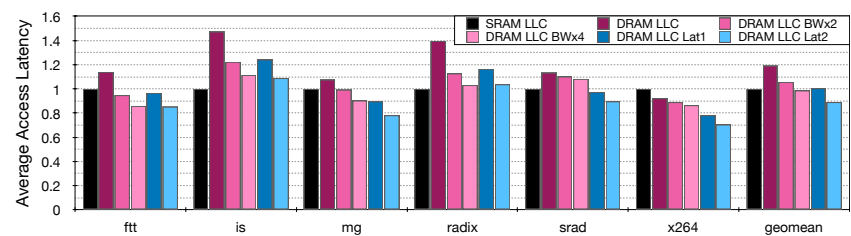

(b) Normalized average memory access latency (from request arrival in memory controller until serviced).

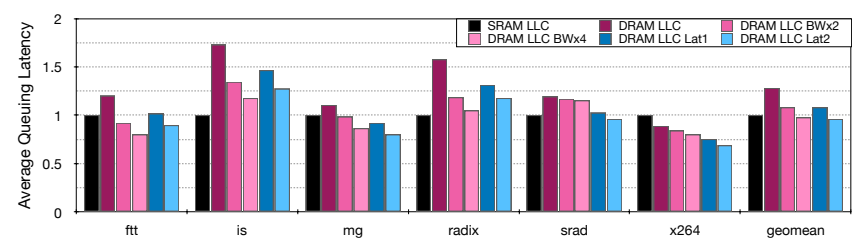

(c) Normalized average queuing latency at the memory controller.

Figure 8: Performance impact of memory bandwidth and latency on HPC workloads

- A fixed area per layer in turn fixates the bandwidth that escapes logic layers. This bandwidth is shared by traffic to all DRAM layers that the logic layer communicates to. Therefore, as we proceed to deeper 3D stacking but with constant layer area, the bandwidth per DRAM layer decreases.

- The conventional placement of memory controllers on a logic layer underneath the DRAM stack will be difficult if each layer shall maintain two independent physical channels, leading to tens of memory controllers on the logic layer. Given the end of Moore's law, area required for the memory controllers might actually require die sizes to increase 2D.

- Since reaching a DRAM layer requires traversing intermediate ones, deeper 3D stacking increases the average distance and thus number of TSVs that are traversed per request.

Consequently, interconnects providing high bandwidth density that is necessary to extract parallelism in applications, low latency, and energy, coupled with DRAM organizations that reduce latency within the DRAM structures, would have have a significantly improved system performance and energy in future 3D integrated systems with 3D-stacked DRAM and also allow performance scaling along with memory capacity. In turn, this can make architectural designs such as DRAM caches [53] and in-memory processing [36] more attractive.

\section{DEEP 3D WITH OPTICS}

\subsection{Enabling Technology}

We propose the use of recently developed vertical optical interconnects (VOI) to overcome the above mentioned design challenges of deep-3D stacking. Optical communication excels in providing high bandwidth density (i.e., bandwidth per area) through wavelengthdivision multiplexing (WDM)-transmitting data on multiple wavelengths in parallel inside the same optical connection ("waveguide"). The utilization of silicon photonics (SiPhs), which denote the technology enabling CMOS-compatible devices for optical communication on-chip, in computing systems has been explored by numerous previous studies $[9,13,14,39,57,106,110,115,116]$. However, no previous studies have explored $\mathrm{SiPhs}$ as a means to improve memory bandwidth within DRAM stacks as a replacement for TSVs, mostly because previously-demonstrated optical TSVs exhibited $\sim 100 \mu \mathrm{m}$ diameters [95]-too big to compete with current TSVs technologies, despite the bandwidth density benefits of WDM.

Recently demonstrated vertical U-shaped couplers and vias significantly change this playing field by offering VOIs with $1-2 \mu \mathrm{m}$ pitches [128], possibly making them a key enabling technology for high-density 3D optically-interconnected DRAM stacks. These VOIs can be fabricated with standard CMOS processes, allow alignment errors within tens of nanometers, and do not require additional packaging steps [128]. Figure 9 illustrates a VOI interconnecting two stacked die layers that consists of two $45 \mathrm{deg}$ reflectors connecting the two silicon waveguide layers and an inter-layer optical via. The transition between the vertical via (1-2 $\mu \mathrm{m}$ thick) and the silicon waveguides (220nm thick) can be made through lateral/vertical tapers with negligible loss [34]. Modulators on the transmitter (tx) side encode bits onto several wavelengths $\left(\lambda_{0} . . \lambda_{x}\right)$ which traverse the waveguides and vertical via and finally filtered and converted back from photons to electrons by microrings filters and photodetectors at the receiver (rx). Connecting to layers higher up the stack is done by implementing a silicon waveguide going through the intermediate layers which merely imposes waveguide propagation loss.

Thickness of 1- $2 \mu \mathrm{m}$ offers dimensions similar to state-of-the-art TSV demonstration on the research-level. However, data can be transmitted on tens of wavelengths through one SiPh link, potentially offering more than an order of magnitude higher bandwidth density than TSVs. Although, for a fair comparison, area for microrings, photodetectors, and $\mathrm{tx} / \mathrm{rx}$ circuitry has to be accounted for, each structure is $\sim 5 \mu \mathrm{m}$ in pitch, allowing for more bandwidth density of optical vias nevertheless. In addition, optical communication is practically distance-independent in terms of energy and latency, which allows to place the $\mathrm{tx} / \mathrm{rx}$ circuitry anywhere on the chips without performance or energy overheads. We provide a detailed area analysis in Section 5.

\subsection{Scaling Memory Capacity of 3D-Stacked DRAM With SiPh}

3.2.1 Design Goals. The bandwidth density properties of VOIs and CMOS compatibility make them a viable candidate to provide a vertical interconnect for deep-3D DRAM stacks superior to TSVs and open up a new design space to be explored. We propose the 


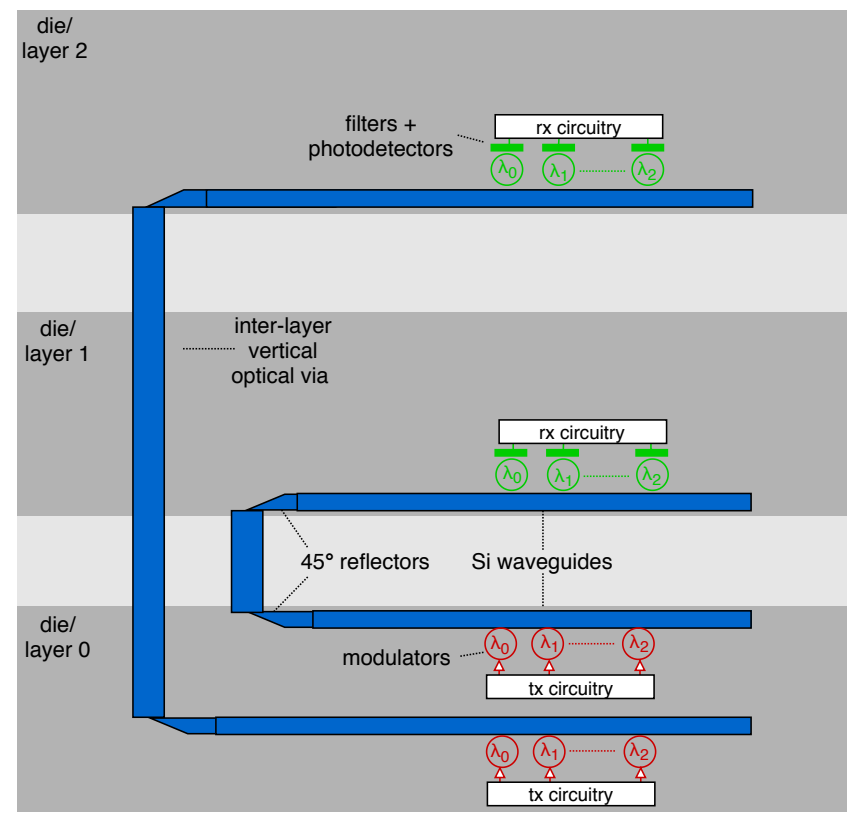

Figure 9: Example silicon vertical optical interconnect (VOI).

design of SiPh DRAM stacks that exploit VOIs to provide an efficient design for future 3D-stacked DRAMs. Aside from overcoming the limitations of TSVs and challenges of deep 3D DRAM, SiPh stacks must be a practical solution from a technology and practicality point of view. Consequently, our design has the following goals:

- Scale bandwidth proportional with the number of DRAM layers while avoiding large area overheads.

- Maintain die sizes (2D) to prevent memory access latency increases due to delay on longer interconnects.

- Design and layout each type of layer (DRAM or logic) uniformly (independently of other layers) to allow for costefficient high-volume manufacturing.

- Provide an efficient solution to overcome the area overheads of large numbers of memory controllers.

3.2.2 Design Structure and Interconnects. Figure 10 illustrates the overall design of a SiPh DRAM stack placed on top of a memory interface (MI) layer (which can be either integrated on the processor die or stacked atop). An external light source provides the light for optical communication to the MI layer and the SiPh layers in the DRAM stack. The SiPh DRAM stack consists of several smaller $\mathrm{SiPh}$ stacks that are duplicated and stacked atop each other.

Each SiPh stack consists of one SiPh layer which accommodates the optical tx and rx circuitry, memory controllers, and TSVs through which it connects to the DRAM dies-the memory controllers and the TSV interface to the DRAM dies is unchanged from the conventional HBM implementation. Therefore, our design uses optical interconnects (VOIs) for communication between the MI layer and the SiPh layers, and conventional electrical TSVs to communication between the memory controllers on the SiPh layers and the DRAM dies/banks. The layout of DRAM banks and TSVs on the DRAM layers is unchanged from conventional DRAM stacks, too, aside from area dedicated to VOIs that must penetrate the DRAM layers to connect to the $\mathrm{SiPh}$ layers above.

3.2.3 Time line of a memory request. The memory interface layer has buffer queues for each $\mathrm{SiPh}$ layer (and in turn $\mathrm{SiPh}$ stack). Upon receiving a memory request from the NoC, the MI layer inspects its address, enqueues it into the queue corresponding to the $\mathrm{SiPh}$ stack, and subsequently forwards the request on the VOI links corresponding to the memory controllers on the $\mathrm{SiPh}$ layer to which the address is mapped. From this point on, the MC enqueues and schedules the memory request based on its scheduling policy, utilizing the same TSV interface (data and CMD/ADDR buses) like in conventional DRAMs. Each MC represents a distinct physical channel and therefore connects to one particular DRAM die.

Once the $\mathrm{MC}$ receives the response from the DRAM die, the MC sends it back to the MI interface over the optical links, which in turn forwards it back to the requester over the NoC.

\subsection{Benefits of SiPh Stacks}

In summary, our hierarchical SiPh stack design is a viable solution towards scalable deep-3D DRAM stacks and overcomes several key challenges of TSV-based DRAM stacks:

Area overheads of uniform chip design. Designing DRAM chips of a stack uniformly (i.e., all DRAM chips are identical to others) allows for high-volume batch processing but leads to a large amount of unused TSV causing unnecessary area overheads that do not scale gracefully with the number of layers. SiPh stacks allow for uniform DRAM chip design without area overheads by dividing the entire stack into sub-stacks and utilizing compact bandwidth-dense optical links to communicate with the sub-stacks. These area savings can be exploited to either place more DRAM cells on each chip or to decrease chip sizes in 2D dimensions to reduce interconnect (and in turn memory) latency. Besides, although $\mathrm{SiPh}$ transceiver impose area overheads compared to electrical interconnects, communication in optics is virtually distance-independent. This enables flexible placement of the transceiver circuitry (e.g., on the edges to alleviate thermal issues, or close to the directory controllers for low latency, etc.) without performance or energy hits.

Area overheads of memory controllers. For a DRAM stack like HBM 2.0, the number of memory controllers (MCs) scales with $M C s=$ layers $\times C H \_p e r \_l a y e r$ for a DRAM stack $\left(C H \_p e r \_l a y e r\right.$ denoting physical channels per layer, in HBM 2.0 two). As the number of layers increases, accommodating all MCs on a single logic layer will become increasingly complex due to the end of feature shrinking and particularly if DRAM die sizes should not be increased significantly horizontally to avoid interconnect delay overheads. Utilizing a light-weight memory interface layer for queuing memory requests and placing MCs on the SiPh layer of a sub-stack provides a scalable solution to increase DRAM capacity vertically without increasing die sizes horizontally. Moreover, our sub-stack design maintains the proximity between the memory controllers and the DRAM banks for low latency and each memory controller on each SiPh layer communicates directly with each controller, therefore typically features like advanced scheduling algorithms or QoS support are not affected by our design.

Distance-agnostic overheads. Photonic communication imposes energy and latency practically independently of distance up 

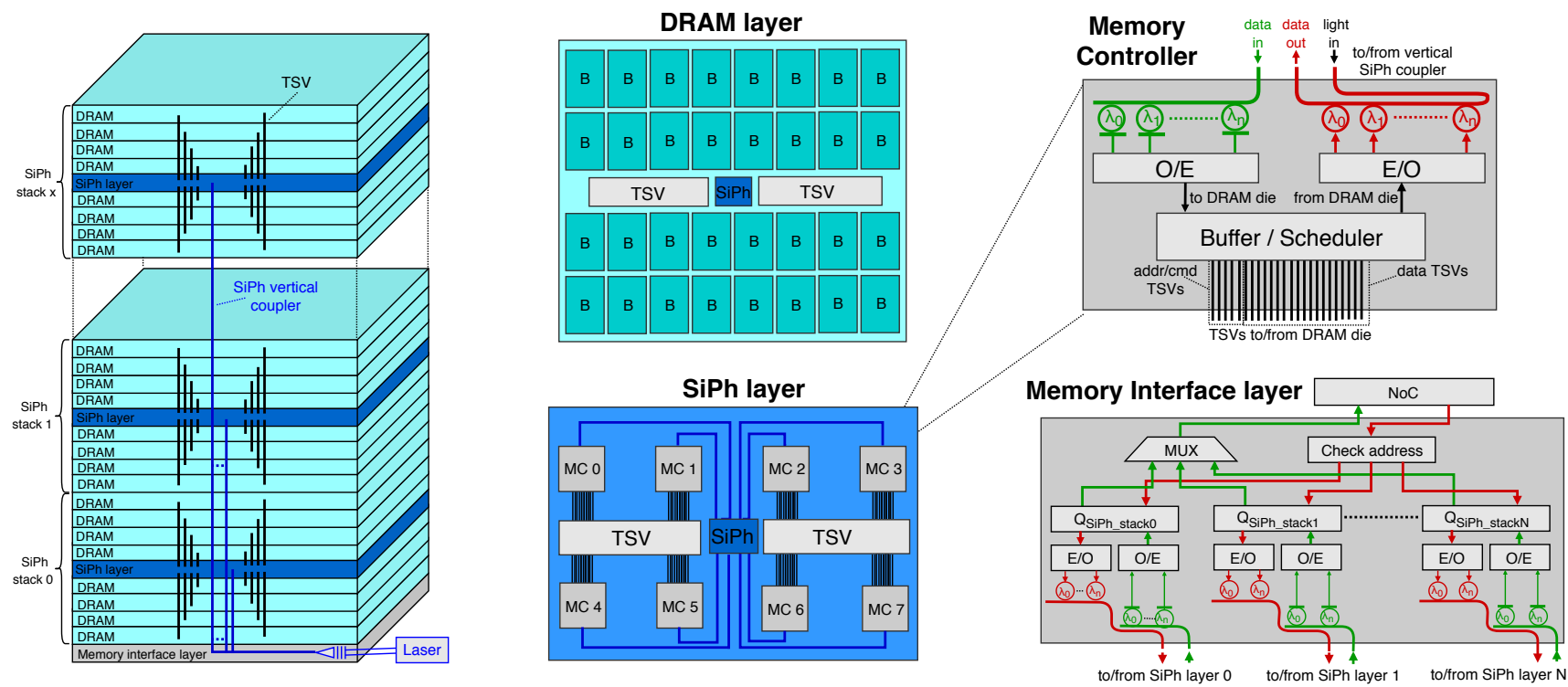

Figure 10: Example design and layout of a SiPh DRAM stack with $x$ number of SiPh stacks placed atop a memory interface layer and its different layers. Each SiPh stack consists of one SiPh layer and an equal number of DRAM layers placed above and below (in this example, 8 DRAM layers, 4 on each side). Memory requests are sent (based on their address) over vertical optical links from the memory interface layers to the memory controllers (MCs) on the SiPh layers, where data is converted back into the electrical domain. The TSV interface between the MCs and the DRAM dies is unchanged from the HBM interface. Similarly, the bank (B) and TSV layout of the DRAM layer is unchanged, except from area for the VOIs which penetrate through each DRAM layer to reach the MCs on the SiPh layers.

to a maximum. Therefore, unlike TSVs and monolithic 3D integration, deep 3D stacks do not penalize traffic to DRAM layers that are far away from logic layers. This means that data in DRAM layers can be distributed more evenly with no communication penalty, reducing the probability of thermal hotspots. This also eliminates memory access latency heterogeneity caused by data placement, that is prevalent in some modern chip multiprocessors [45]. Likewise, logic layers that typically have higher heat dissipation can be placed farther apart from each other with no adverse communication impact. In addition, photonic communication can increase the bandwidth escaping from logic layers for the same area overhead, thus the bandwidth between logic layers as well as the bandwidth between logic and DRAM layers.

The following section provides a detailed analysis of the scalability and resources requirements of $\mathrm{SiPh}$ stacks as well as a detailed comparison to TSV based stacks.

\section{METHODOLOGY}

We provide a detailed analytic analysis of the $\mathrm{SiPh}$ resource requirements and scalability of SiPh stacks as well as a comparison to state-of-the-art TSV based stacks in terms of area, power consumption, and performance.

\subsection{Performance Modeling}

We use Gem5 [16] in full-system mode (booted with Linux kernel version 16.4) with Garnet2.0 [3] for modeling the Network-onchip and Ruby (from GEMS [76]) for modeling the cache hierarchy.
We evaluate our designs with a wide range of applications from several different benchmark suites, i.e. PARSEC 3.0 [124], Splash2x [124] (both sim-large input size), Rodinia [26], and NAS Parallel Benchmark suite (NBP) [11] (C input size). We chose applications with a variety of bandwidth and latency characteristics to study the performance impact of our SiPh stacks in different use-case scenarios.

\subsection{Power Modeling}

4.2.1 Electrical Components. We estimate energy consumption of the electrical circuitry of the transceiver (i.e., SERDES and drivers) based on demonstrated transceiver technologies in $65 \mathrm{~nm}$ CMOS scaled down to $28 \mathrm{~nm}$ using SPICE models [67, 123]. We use DSENT [109] to estimate the power consumption of the buffers and crossbars in the memory interface layer, also for a $22 \mathrm{~nm}$ technology node. An overview of the main components in our Gem5 simulation environment is shown in Figure 11.

4.2.2 Photonic Components. We model power consumption of $\mathrm{SiPh}$ interconnects based on the reported device loss values and receiver sensitivity of demonstrated transceiver technologies in 65nm CMOS (including clock generation/recovery and microring tuning) scaled down with SPICE models to $28 \mathrm{~nm}[67,123]$ with laser efficiency of commercially-available comb lasers [38]. Loss of VOI corresponds to regular waveguide loss when vertically penetrating dies and $1.3 \mathrm{~dB}$ when coupled horizontally into a die [128]. We performed power modeling our all $\mathrm{SiPh}$ with our power modeling tool that 


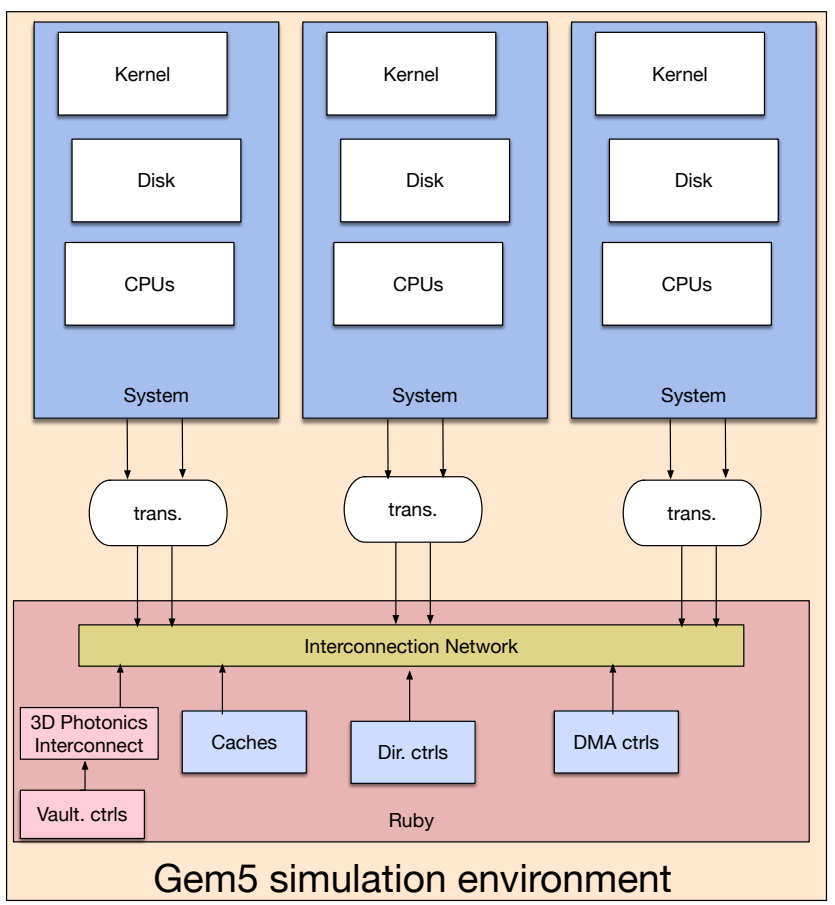

Figure 11: 3D interconnect Power Model integration in Gem5.

Table 4: SiPh device parameters.

\begin{tabular}{llll}
\hline Parameter & Value & Parameter & Value \\
\hline Optical Fiber & $5 \mathrm{e}-6 \mathrm{~dB} / \mathrm{cm}$ & Photodetector loss & $0.1 \mathrm{~dB}$ \\
Modulator Insertion loss & $1 \mathrm{~dB}$ & Power Margin & $3 \mathrm{~dB}$ \\
Waveguide loss & $0.5 \mathrm{~dB} / \mathrm{cm}$ & Filter through loss & $0.1 \mathrm{~dB}$ \\
Filter drop loss & $1.5 \mathrm{~dB}$ & Receiver Sensitivity & $-17 \mathrm{dBm}$ \\
Coupler: Fiber-to-Package & $3 \mathrm{~dB}$ & Laser efficiency & $14 \%$ \\
Coupler: Package-to-Chiplet & $0.5 \mathrm{~dB}$ & & \\
\hline
\end{tabular}

reads in the simulation output (i.e., link traversals, simulation time, etc.) of gem5.

\subsection{Area Modeling}

We compare the area requirements of VOIs and TSVs to quantify the area benefits of VOIs. For a fair comparison, we include area for the transmitter and receiver circuitry of the SiPh components. However, as discussed earlier, communication is distance-independent in optics which allows the transceiver circuitry anywhere on the memory interface layer and $\mathrm{SiPh}$ layer, allowing for more layout flexibility.

We estimate area of the electronic components in the memory interface layer and SiPh layer with DSENT's 22nm technology node. We assume a $2 \mu \mathrm{m}$ pitch for the VOI [123], $5 \mu$ pitch for the waveguides, and microring resonators with $5 \mu \mathrm{m}$ radii. For TSV pitches, we vary the size based on the demonstrated TSV technologies listed in Table 1.

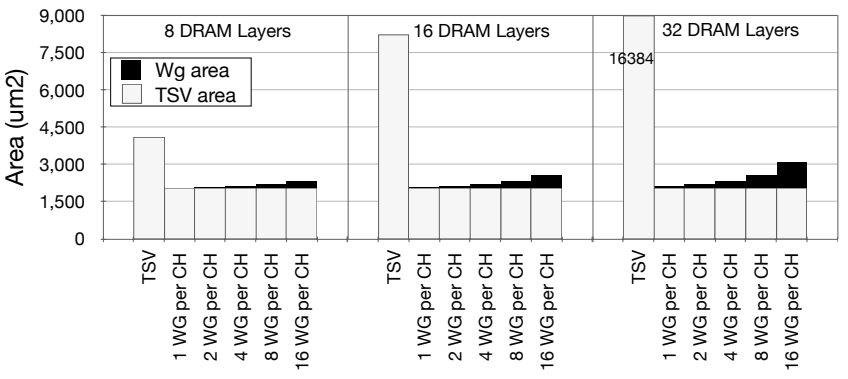

(a) $2 \mu \mathrm{m}$ TSV Pitch

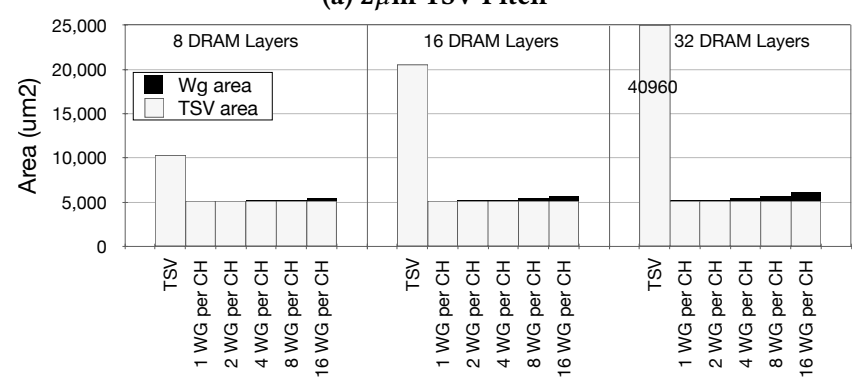

(b) $5 \mu \mathrm{m}$ TSV Pitch

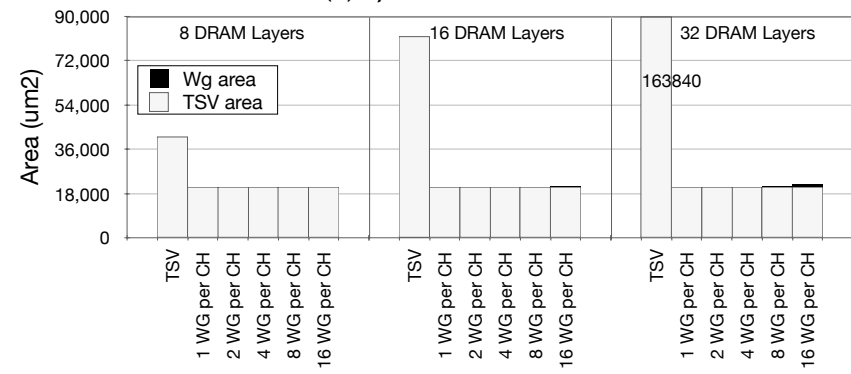

(c) $20 \mu \mathrm{m}$ TSV Pitch

Figure 12: Area footprint of vertical interconnects on each DRAM die for 8, 16, and 32 high stacks. SiPh stacks consist of substacks with 8 DRAM layers each. TSV denotes a conventional DRAM stack with just TSVs, while the optical interconnect to the $\mathrm{SiPh}$ layer in SiPh stacks is reported for a varying number of waveguides per physical DRAM channel.

\section{EVALUATION RESULTS}

\subsection{Area Analysis}

Figure 12 compares the vertical interconnect area requirements of our SiPh substack approach with conventional electrical TSVs (for $2 \mu \mathrm{m}, 5 \mu \mathrm{m}$, and $20 \mu \mathrm{m}$ pitch) for providing the same aggregate DRAM bandwidth. We consider stacks of different heights $(8,16$, and 32 DRAM layers) to investigate the impact of vertical interconnect area on current and future DRAM stacks. Moreover, we study the area of VOIs with spacial-division multiplexing (SDM), a well-known approach to reduce laser power by reducing the number of wavelengths per channel. Area requirements for vertical interconnects directly determine the area on each DRAM layer that will have to be dedicated to the interconnect and can thus not be used for memory cells. 
We make three key observations: First, the small pitch of vertical SiPh waveguides and their ability to provide high bandwidth density with several wavelengths per waveguide will make the overall area requirements insignificant compared to what would be needed if only TSVs were available for interconnection. Second, as the number of DRAM layers per stack increases, these benefits will become increasingly evident, and area requirements would be cut in half for SiPh vertical links. Third, using spacial-division multiplexing to decrease the number of wavelengths per channel has no significant impact on the area savings of SiPhs, even for 16 waveguides per DRAM channel. SDM is therefore an approach that could be heavily used to cut down laser power costs without concerns for exorbitant area overheads.

Figure 13 shows area breakdowns of the vertical interconnects in DRAM stacks with 8, 16, and 32 layers including area for SiPh transceiver (trx) circuitry on the network interface and SiPh layers. Generally, although SiPh trx circuitry is relatively large compared to CMOS, overall area requirements are only higher than stacks based on electrical TSVs if we assume aggressive TSV pitches of $=<5 \mu \mathrm{m}$ and DRAM stacks with $=<16$ layers. As we look ahead, as attaining $=<5 \mu \mathrm{m}$ TSV pitches at very large scale to support tens of DRAM layers will be questionable, our analysis showing that the overall area requirements of VOIs are not high compared to $=<5 \mu \mathrm{m}$ TSV pitches is highly encouraging. This is particularly the case as trx area is the predominant contributor to the overall area of VOIs, meaning that large area savings are attained on the actual DRAM layers, allowing for higher DRAM storage cell counts on each layer.

\subsection{Performance Results}

Accessing the DRAM with optical interconnects inevitably incurs some latency overhead compared to electrical TSVs due to the electrical-to-optical-to-electrical (E-O-E) conversion step that is required for both the request and response to/from the DRAM. Traversing the switch on the network interface incurs additional latency as well. However, traversing on-chip switches and E-O-E conversion can typically be executed within one core clock cycle each. We hypothesize that these latency overheads are insignificant to overall system performance as they are a small fraction of the time it takes, for instance, to access an open DRAM bank.

To confirm this assumption, we perform full-system simulation of a 16-core system with both VOIs and and electrical TSVs, the results of which are shown in Figure 14 that depicts the total application execution for over a range of different HPC workloads. We observe that the highest execution time overhead (for the 'is' workload) is $5 \%$ for using VOIs. However, the vast majority of applications do not show any significant increase in execution time, leading to an average of $2 \%$ higher execution time.

It is worth noting that latency for VOIs remains practically constant even for more DRAM layers. In contrast, average latency using TSVs will grow proportionally with the number of DRAM layers. Therefore, future deep 3D stacks will experience a latency reduction for VOIs that we do not notice in shallow stacks.

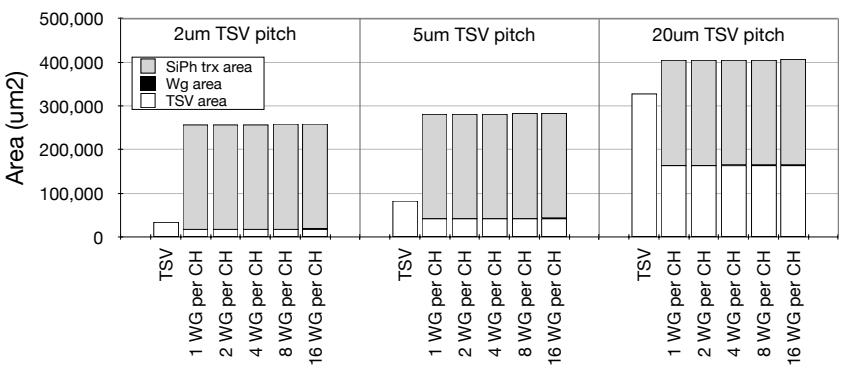

(a) 8 DRAM Layers

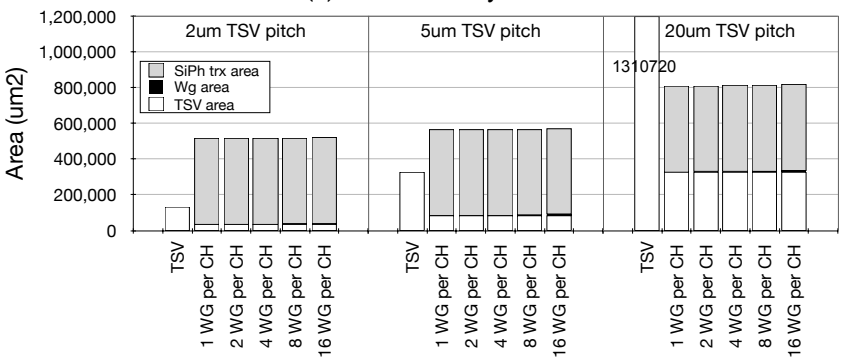

(b) 16 DRAM Layers

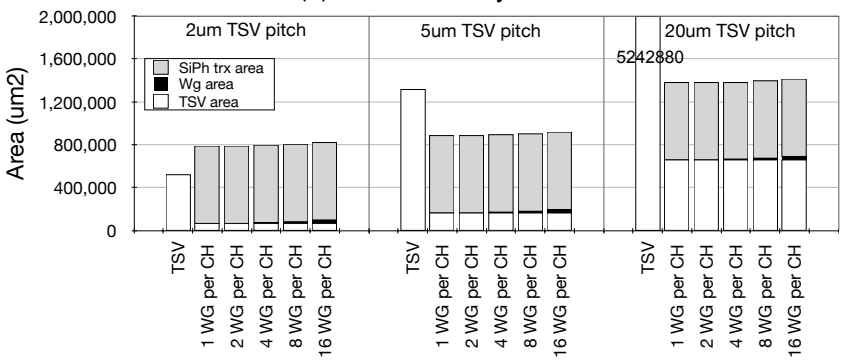

(c) 32 DRAM Layers

Figure 13: Total vertical interconnect area of an entire stack, including SiPh transceiver (trx) circuitry on the memory interface and SiPh layers.

\subsection{Energy Results}

In terms of DRAM access energy, conventional DRAM with electrical TSV and our proposed SiPh DRAM layout are similar for activation energy and on-die data movements. However, the I/O energy in each case is different because they use different implementations. In conventional DRAM ${ }^{4}$, the I/O requires $800 \mathrm{fJ} / \mathrm{bit}$ [90] which is expected to increase as the height of DRAM stacks increases. For a SiPh stack, using parameters shown in 4 to match realistic current technologies, the total I/O energy (including laser, SERDES, modulation circuitry) would be $760 \mathrm{fJ} / \mathrm{bit}$.

Figure 15 illustrates a comparison of DRAM I/O energy between a conventional DRAM stack interconnected electrically with TSVs against SiPh DRAM stacks across a variety of HPC applications. The power values correspond to an 8-layer-high stack, where we assume one $\mathrm{SiPh}$ layer above/below four DRAM layers in the SiPh case with a stack height of an 8-layer HBM (as described in Section 2). As shown, the VOI DRAM stack reduces DRAM access energy

${ }^{4}$ Scaled down to $28 \mathrm{~nm}$ to provide a fair comparison between the electrical and optical designs. 


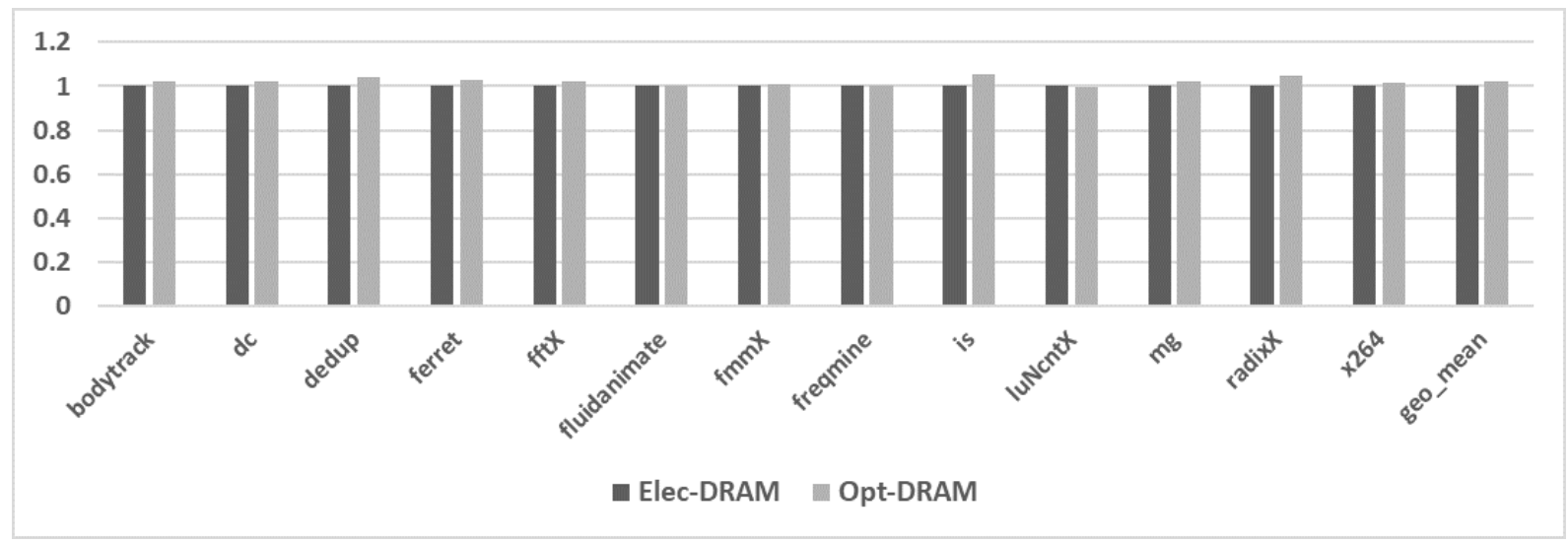

Figure 14: Execution time for the electrical and optical DRAM architectures (normalized to electrical design)

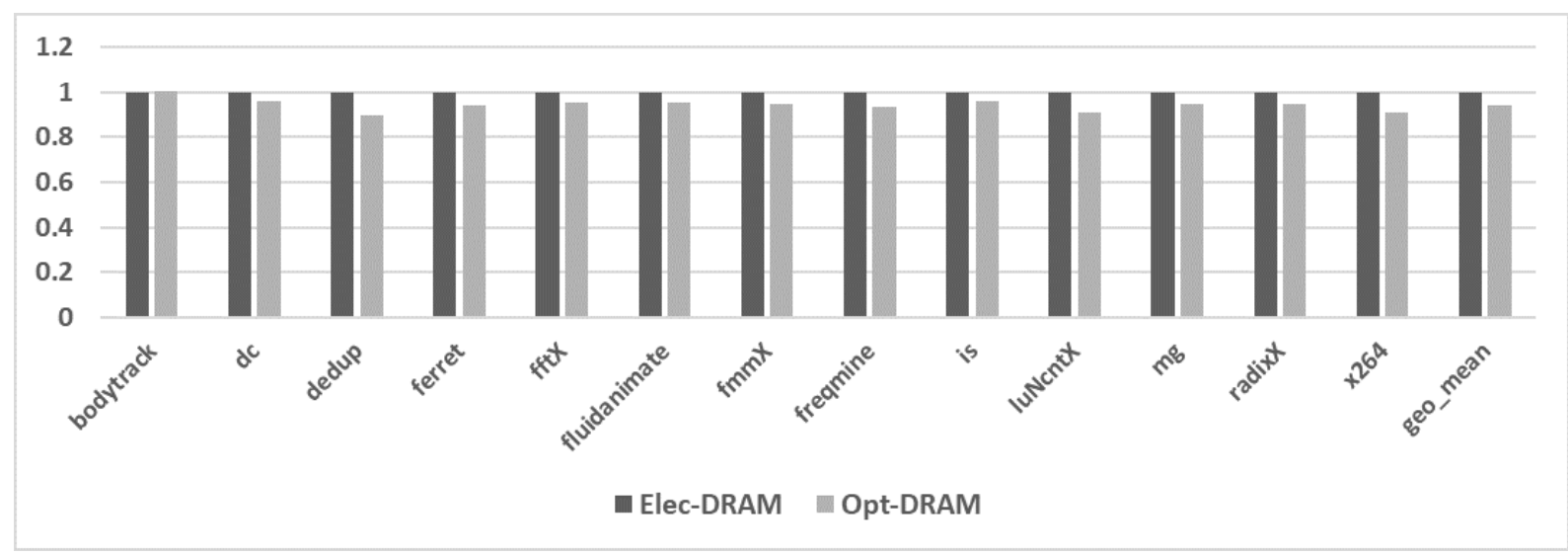

Figure 15: DRAM I/O Energy for the electrical and optical DRAM architectures (normalized to electrical design)

by approximately 5\%, which will further increase with deeper 3D stacks. That is because with electrical designs, communication to any DRAM layer has to traverse intermediate layers. Therefore, deeper 3D integration will increase the average energy of communication proportionally to distance.

\subsection{Discussion}

The results of our study can be summarized into three key findings that could be crucial for the future of efficient deep-3D DRAM architectures:

(i) Utilizing VOIs in combination with our hierarchical 3D DRAM stacks leads to tremendous savings ( $2 \times$ in our setting) in interconnect area which can in turn be utilized to integrate more storage cells within the same die dimensions leading to higher memory density. Although SiPh interface circuitry has area footprint overheads compared to TSVs, these area overheads only have to be paid on the few interface layers. This offers a much more scalable-and thereby potentially enabling-approach for future deep-3D memories with tens of die layers.

(ii) Despite the latency overheads of SiPhs for electrical-tooptical signal conversion, we observe that these overheads are insignificant in comparison to DRAM access latency. Consequently, our simulation results indicate negligible performance hits. Given that DRAM latency is an overhead that is notoriously hard to overcome without significant cost and/or power overheads, and alternative memory technologies (such as non-volatile memories) do not exhibit noticeable decreases in latency, we hypothesize that the conversion latency overheads of $\mathrm{SiPh}$ are unlikely to play a significant role any time soon.

(iii) $\mathrm{SiPh}$ can provide lower energy for some design points despite E-O-E conversions because in electric DRAM stacks communication energy is proportional to distance. Therefore, this will increase the energy overhead with deeper 3D integration. In contrast, the energy for communication for $\mathrm{SiPh}$ is practically independent of distance for on-chip distances. In addition, distance-independent communication energy allows data to be placed more uniformly across DRAM layers to alleviate thermal hotspots without increasing DRAM access latency. This also eliminates memory access latency heterogeneity caused by data placement, that is prevalent in some modern chip multiprocessors [45]. Finally, logic layers can be placed farther apart to alleviate thermal hotspots with no noticeable latency and energy penalties. 
Based on these findings we believe that SiPh might be a key enabling technology to provide deep memory stacks with tens or hundreds of DRAM layers due to drastically smaller area combined with a small impact to latency and energy that will only improve with deeper 3D stacking.

\section{RELATED WORK}

\subsection{Wireless Vertical Interconnects}

Wireless (or contactless) vertical links based on AC coupling have recently been proposed (at the research-level) as a substitute for TSVs $[70,83,84]$. Wireless inter-layer communication facilitates the development of 3D systems by employing passive elements within each layers of a 3D stack that can be manufactured using conventional 2D processes [93]. This allows seamless heterogeneous integration of disparate technologies in the same package [96] as opposed to relying on additional processing steps for TSVs (e.g. TSV etching and copper filling) [108]. Wireless links thus hold the potential of cheaper communication than wired schemes. While both inductive and capacitive contact-less communication are compatible with conventional CMOS processes [10, 37, 51, 82], inductive links offer superior performance [93]. Moreover, inductive links provide high versatility as they do not limit 3D systems to face-toface integration, but also provide support for face-up/down and back-to-back bonding [47].

Contact-less-in particular inductive-links can be used to form 3D on-chip network topologies [64] and are a promising technology in terms of manufacturing, but can currently not compete with TSVs in terms of bandwidth density. A recent study shows that individual inductive links can match the maximum performance of TSVs (with $20 \mu \mathrm{m}$ diameter) but provide significantly less bandwidth density due to the area taken up by the necessary transceiver circuitry [94 $]^{5}$. Trading-off area for manufacturing complexity might be reasonable for 3D systems with relatively low inter-layer bandwidth; however, this does not apply to systems with 3D-stacked DRAM that rely on the relatively high bandwidth density of TSVs to satisfy the large memory bandwidth demands of various modern workloads.

\subsection{Optics in 3D}

The promise of on-chip optics in 3D integration has been acknowledged [8], with work to design a suitable bus-based or point-topoint network topology and communication protocol [18, 19, 55], a hybrid electrical-optical network [4], or a new router architecture [40]. Further work has used optics to provide reconfigurability in a 3D on-chip network [86], thermal-aware routing [121], as well as design an all-optical on-chip network with wavelength division multiplexing [42]. At the same time, several open questions remain [117]. The promise of 3D optics in providing higher bandwidth density has also been briefly acknowledged [89, 98].

Our study advances the state of the art by quantifying the impact of on-chip photonics in deep 3D integration with multiple logic and memory layers. We demonstrate how many memory layers we can reasonably integrate with TSVs, and how this limit increases with the bandwidth density of 3D photonics. In turn, we discuss the

\footnotetext{
${ }^{5}$ The total area, however, is comparable since inductive links primarily occupy in terconnect area and only silicon area for the transceivers, allowing logic gates to be placed above/below, which is not the case for TSVs [85]
}

architectural implications to better take advantage of photonics, as well as the impact of photonic latencies in memory and logic layer placement, which affects thermal hotspots.

\section{CONCLUSION}

Deep 3D stacking is a promising avenue towards preserving computational performance scaling in the near and distant future. However, conventional integration technologies such as monolithic 3D integration and TSVs threaten to constrain the practical number of DRAM layers due to bandwidth density and energy to reach distant 3D layers. In this paper, we argue for the promise of vertical $\mathrm{SiPh}$ interconnects as an enabling technology due to their higher bandwidth density and their near distance-agnostic energy and latency overheads. Based on this observation, we explore the benefits and architectural implications of SiPh vertical interconnects in terms of area, power, and performance. Then, we propose a hierarchical approach to stacking 3D DRAM to tens of layers by utilizing substacks which are optically-interconnected to a memory interface on the processor die. Our simulation results show that our design, despite requiring electrical-to-optical conversion, exhibits negligible performance overhead and slightly reduces power consumption over a range of HPC workloads compared to conventional TSVbased DRAM stacks, while halving interconnect area requirements for the same layer-to-layer bandwidth.

\section{ACKNOWLEDGMENTS}

This work was supported by the Director, Office of Science, of the U.S. Department of Energy under Contract No. DE- AC02$05 \mathrm{CH} 11231$.

\section{REFERENCES}

[1] MonolithIC 3D. February, 2013. MonolithIC 3D ICs. http://www.monolithic3d. com/-papers-presentations-and-patents.html.

[2] MonolithIC 3D. October, 2015. MonolithIC 3D: The most effective path for future IC scaling. http://www.monolithic3d.com/uploads/6/0/5/5/6055488/09_ 3d_integration_2013.pdf.

[3] Niket Agarwal, Tushar Krishna, Li-Shiuan Peh, and Niraj K Jha. 2009. GARNET: A detailed on-chip network model inside a full-system simulator. In 2009 IEEE international symposium on performance analysis of systems and software. IEEE, 33-42.

[4] A. B. Ahmed and A. B. Abdallah. 2013. PHENIC: silicon photonic 3D-networkon-chip architecture for high-performance Heterogeneous many-core systemon-chip. In 14th International Conference on Sciences and Techniques of Automatic Control Computer Engineering - STA'2013. 1-9. https://doi.org/10.1109/STA.2013. 6914696

[5] Jung Ho Ahn, Norman P Jouppi, Christos Kozyrakis, Jacob Leverich, and Robert S Schreiber. 2012. Improving system energy efficiency with memory rank subsetting. ACM Transactions on Architecture and Code Optimization (TACO) 9, 1 (2012), 4.

[6] Jung Ho Ahn, Jacob Leverich, Robert Schreiber, and Norman P Jouppi. 2009. Multicore DIMM: An energy efficient memory module with independently controlled DRAMs. IEEE Computer Architecture Letters 8, 1 (2009), 5-8.

[7] Tong An, Fei Qin, Wei Wu, Daquan Yu, Lixi Wan, and Jun Wang. 2012. Effects of via pitch on silicon stress in TSV interposer. In 2012 13th International Conference on Electronic Packaging Technology \& High Density Packaging. IEEE, 600-605.

[8] and and. 2017. Designs of 3D mesh and torus optical Network-on-Chips: Topology, optical router and routing module. China Communications 14, 5 (May 2017), 17-29. https://doi.org/10.1109/CC.2017.7942191

[9] Yasuhiko Arakawa, Takahiro Nakamura, Yutaka Urino, and Tomoyuki Fujita. 2013. Silicon photonics for next generation system integration platform. IEEE Communications Magazine 51, 3 (2013), 72-77.

[10] Myat Thu Linn Aung, Eric Lim, Takefumi Yoshikawa, and Tony Tae-Hyoung Kim. 2014. A 3-Gb/s/ch simultaneous bidirectional capacitive coupling transceiver for 3DICs. IEEE Transactions on Circuits and Systems II: Express Briefs 61, 9 (2014), 706-710. 
[11] David H Bailey. 2011. NAS parallel benchmarks. Encyclopedia of Parallel Computing (2011), 1254-1259.

[12] Adeel A Bajwa, SivaChandra Jangam, Saptadeep Pal, Niteesh Marathe, Tingyu Bai, Takafumi Fukushima, Mark Goorsky, and Subramanian S Iyer. 2017. Heterogeneous Integration at Fine Pitch (âL'd $10 \mu \mathrm{m}$ ) Using Thermal Compression Bonding. In 2017 IEEE 67th Electronic Components and Technology Conference (ECTC). IEEE, 1276-1284.

[13] Scott Beamer, Krste Asanović, Christopher Batten, Ajay Joshi, and Vladimir Stojanović. 2009. Designing multi-socket systems using silicon photonics. In Proceedings of the 23rd international conference on Supercomputing (ICS). ACM $521-522$.

[14] Scott Beamer, Chen Sun, Yong-Jin Kwon, Ajay Joshi, Christopher Batten, Vladimir Stojanović, and Krste Asanović. 2010. Re-architecting DRAM memory systems with monolithically integrated silicon photonics. In ACM SIGARCH Computer Architecture News, Vol. 38. ACM, 129-140.

[15] Eric Beyne. 2016. The 3-D interconnect technology landscape. IEEE Design \& Test 33, 3 (2016), 8-20.

[16] Nathan Binkert, Bradford Beckmann, Gabriel Black, Steven K Reinhardt, Al Saidi, Arkaprava Basu, Joel Hestness, Derek R Hower, Tushar Krishna, Somayeh Sardashti, et al. 2011. The gem 5 simulator. ACM SIGARCH Computer Architecture News 39, 2 (2011), 1-7.

[17] L Brunet, P Batude, C Fenouillet-Beranger, P Besombes, L Hortemel, F Ponthenier, B Previtali, C Tabone, A Royer, C Agraffeil, et al. 2016. First demonstration of a CMOS over CMOS 3D VLSI CoolCubeâĎc integration on $300 \mathrm{~mm}$ wafers. In 2016 IEEE Symposium on VLSI Technology. IEEE, 1-2.

[18] Q. Cai, W. Hou, C. Yu, P. Han, L. Zhang, and L. Guo. 2014. Design and OPNET implementation of routing algorithm in 3D optical network on chip. In 2014 IEEE/CIC International Conference on Communications in China (ICCC). 112-115. https://doi.org/10.1109/ICCChina.2014.7008253

[19] L. P. Carloni, P. Pande, and Y. Xie. 2009. Networks-on-chip in emerging interconnect paradigms: Advantages and challenges. In 2009 3rd ACM/IEEE International Symposium on Networks-on-Chip. 93-102. https://doi.org/10.1109/NOCS.2009. 5071456

[20] Cathal Cassidy, Jochen Kraft, Sara Carniello, Frederic Roger, Hajdin Ceric, An derson Pires Singulani, Erasmus Langer, and Franz Schrank. 2012. Through silicon via reliability. IEEE Transactions on Device and Materials Reliability 12, 2 (2012), 285-295.

[21] Kyungwook Chang, Abhishek Koneru, Krishnendu Chakrabarty, and Sung Kyu Lim. 2017. Design automation and testing of monolithic 3D ICs: Opportunities, challenges, and solutions. In 2017 IEEE/ACM International Conference on Computer-Aided Design (ICCAD). IEEE, 805-810.

[22] Kevin K Chang, Abhijith Kashyap, Hasan Hassan, Saugata Ghose, Kevin Hsieh, Donghyuk Lee, Tianshi Li, Gennady Pekhimenko, Samira Khan, and Onur Mutlu. 2016. Understanding latency variation in modern DRAM chips: Experimental characterization, analysis, and optimization. In ACM SIGMETRICS Performance Evaluation Review, Vol. 44. ACM, 323-336.

[23] Kevin K Chang, Prashant J Nair, Donghyuk Lee, Saugata Ghose, Moinuddin K Qureshi, and Onur Mutlu. 2016. Low-cost inter-linked subarrays (LISA): Enabling fast inter-subarray data movement in DRAM. In 2016 IEEE International Symposium on High Performance Computer Architecture (HPCA). IEEE, 568-580.

[24] Niladrish Chatterjee, Manjunath Shevgoor, Rajeev Balasubramonian, Al Davis, Zhen Fang, Ramesh Illikkal, and Ravi Iyer. 2012. Leveraging heterogeneity in DRAM main memories to accelerate critical word access. In 2012 45th Annual IEEE/ACM International Symposium on Microarchitecture. IEEE, 13-24.

[25] FX Che, L Xie, ZH Chen, and Sunil Wickramanayaka. 2017. Study on warpage and stress of TSV wafer with ultra-fine pitch vias for high density chip stacking. In 2017 IEEE 19th Electronics Packaging Technology Conference (EPTC). IEEE, 1-7.

[26] Shuai Che, Michael Boyer, Jiayuan Meng, David Tarjan, Jeremy W Sheaffer, SangHa Lee, and Kevin Skadron. 2009. Rodinia: A benchmark suite for heterogeneous computing. In 2009 IEEE International Symposium on Workload Characterization (IISWC). Ieee, 44-54.

[27] Jeongdong Choe. June, 2017. Samsung 18nm DRAM cell integration: QPT and higher uniformed capacitor high-k dielectrics. https://techinsights.com/abouttechinsights/overview/blog/samsung-18-nm-dram-cell-integration-qpt-andhigher-uniformed-capacitor-high-k-dielectrics/.

[28] R. Clapp, M. Dimitrov, K. Kumar, V. Viswanathan, and T. Willhalm. 2015. Quantifying the Performance Impact of Memory Latency and Bandwidth for Big Data Workloads. In 2015 IEEE International Symposium on Workload Characterization. 213-224. https://doi.org/10.1109/IISWC.2015.32

[29] Kristof Croes, Joke De Messemaeker, Yunlong Li, Wei Guo, Olalla Pedreira, Vladimir Cherman, Michele Stucchi, Ingrid De Wolf, and Eric Beyne. 2016 Reliability challenges related to TSV integration and 3D stacking. IEEE Design \& Test of Computers 1 (2016), 1-1.

[30] Alexandre Ayres de Sousa. 2017. 3D Monolithic Integration: performance, Power and Area Evaluation for 14nm and beyond. Ph.D. Dissertation. Université Grenoble Alpes.
[31] Ashish Dembla, Yue Zhang, and Muhannad S Bakir. 2012. High aspect ratio TSVs in micropin-fin heat sinks for 3D ICs. In 2012 12th IEEE International Conference on Nanotechnology (IEEE-NANO). IEEE, 1-6.

[32] Jaber Derakhshandeh, Inge De Preter, Carine Gerets, Lin Hou, Nancy Heylen, Eric Beyne, Gerald Beyer, John Slabbekoorn, Vikas Dubey, Anne Jourdain, et al. 2016. 3D stacking using bump-less process for sub $10 \mathrm{um}$ pitch interconnects. In 2016 IEEE 66th Electronic Components and Technology Conference (ECTC). IEEE, $128-133$.

[33] Jaber Derakhshandeh, Lin Hou, Inge De Preter, Carine Gerets, Samuel Suhard, Vikas Dubey, Geraldine Jamieson, Fumihiro Inoue, Tomas Webers, Pieter Bex, et al. 2016. Die to wafer 3D stacking for below 10um pitch microbumps. In 2016 IEEE International 3D Systems Integration Conference (3DIC). IEEE, 1-4.

[34] Po Dong, Ting-Chen Hu, Tsung-Yang Liow, Young-Kai Chen, Chongjin Xie, Xianshu Luo, Guo-Qiang Lo, Rose Kopf, and Alaric Tate. 2014. Novel integration technique for silicon/III-V hybrid laser. Optics express 22, 22 (2014), 26854-26861.

[35] Pete Ehrett, Vidushi Goyal, Opeoluwa Matthews, Reetuparna Das, Todd Austin, and Valeria Bertacco. 2017. Analysis of Microbump Overheads for 2.5 D Disintegrated Design. UMich. Ann Arbor Tech. Rep. CSE-TR-002-17 (2017).

[36] D. G. Elliott, M. Stumm, W. M. Snelgrove, C. Cojocaru, and R. Mckenzie. 1999. Computational RAM: implementing processors in memory. IEEE Design Test of Computers 16, 1 (Jan 1999), 32-41. https://doi.org/10.1109/54.748803

[37] Alberto Fazzi, Luca Magagni, Mauro Mirandola, Barbara Charlet, LÉa Di Cioccio, Erik Jung, Roberto Canegallo, and Roberto Guerrieri. 2007. 3-D capacitive interconnections for wafer-level and die-level assembly. IEEE fournal of SolidState Circuits 42, 10 (2007), 2270-2282.

[38] Innolume GmbH. 2013. Fiber coupled comb laser diode @ 1310nm. https: //www.innolume.com/_pdfs/Comb/LD-1310-COMB-8.pdf.

[39] Paolo Grani, Roberto Proietti, Venkatesh Akella, and SJ Ben Yoo. 2017. Design and Evaluation of AWGR-Based Photonic NoC Architectures for 2.5 D Integrated High Performance Computing Systems. In IEEE International Symposium on High Performance Computer Architecture (HPCA). IEEE, 289-300.

[40] H. Gu and J. Xu. 2009. Design of 3D Optical Network on Chip. In 2009 Symposium on Photonics and Optoelectronics. 1-4. https://doi.org/10.1109/SOPO.2009. 5230071

[41] Wei Guo, Victor Moroz, Geert Van der Plas, M Choi, Augusto Redolfi, L Smith, Geert Eneman, Stefaan Van Huylenbroeck, PD Su, Andrej Ivankovic, et al. 2013. Copper through silicon via induced keep out zone for 10nm node bulk FinFET CMOS technology. In 2013 IEEE International Electron Devices Meeting. IEEE, $12-8$.

[42] P. K. Hamedani, N. E. Jerger, and S. Hessabi. 2014. OuT: A low-power optical Network-on-Chip. In 2014 Eighth IEEE/ACM International Symposium on Networks-on-Chip (NoCS). 80-87. https://doi.org/10.1109/NOCS.2014.7008765

[43] Cheng-chieh Hsieh, Hung-An Teng, Shang-Yun Hou, and Shin-puu Jeng. 2015. Through silicon via keep out zone formation method and system. US Patent $9,054,166$.

[44] Tzu-Chien Hsu, Po-Chih Chang, Chung-Chih Wang, Yiu-Hsiang Chang, TsuenSung Chen, Yu-Chen Hsin, Jui-Chin Chen, Yuan-Chang Lee, Shang-Chun Chen, Jen-Chun Wang, et al. 2016. Backside-TSV process development and integration for $23 \mathrm{um}$ small size TSV. In 2016 11th International Microsystems, Packaging, Assembly and Circuits Technology Conference (IMPACT). IEEE, 273-276.

[45] Y. Huang and D. Li. 2017. Performance Modeling for Optimal Data Placement on GPU with Heterogeneous Memory Systems. In 2017 IEEE International Conference on Cluster Computing (CLUSTER). 166-177. https://doi.org/10.1109/ CLUSTER.2017.42

[46] F Inoue, H Philipsen, A Radisic, S Armini, Y Civale, P Leunissen, and S Shingubara. 2012. Novel seed layer formation using direct electroless copper deposition on ALD-Ru layer for high aspect ratio TSV. In 2012 IEEE International Interconnect Technology Conference. IEEE, 1-3.

[47] Hiroki Ishikuro, Noriyuki Miura, and Tadahiro Kuroda. 2007. Wideband inductive-coupling interface for high-performance portable system. In 2007 IEEE Custom Integrated Circuits Conference. IEEE, 13-20.

[48] ITRS. [n.d.]. International Technology Roadmap for Semiconductors 2.0. http: //www.itrs2.net

[49] JEDEC. 2015. High bandwidth memory (HBM) DRAM. https://www.jedec.org/ standards-documents/docs/jesd235a. [Online; accessed 03-14-2018]

[50] JEDEC. 2018. JEDEC Updates Groundbreaking High Bandwidth Memory (HBM) Standard. https://www.jedec.org/news/pressreleases/jedec-updatesgroundbreaking-high-bandwidth-memory-hbm-standard- 0 .

[51] Min-Ki Jeon and Changsik Yoo. 2017. A simultaneously bidirectional inductively coupled link in a $0.13-\mu \mathrm{m}$ CMOS technology. International fournal of Circuit Theory and Applications 45, 4 (2017), 515-529.

[52] Djordje Jevdjic, Gabriel H Loh, Cansu Kaynak, and Babak Falsafi. 2014. Unison cache: A scalable and effective die-stacked DRAM cache. In Proceedings of the 47th Annual IEEE/ACM International Symposium on Microarchitecture. IEEE Computer Society, 25-37.

[53] Djordje Jevdjic, Stavros Volos, and Babak Falsafi. 2013. Die-stacked DRAM Caches for Servers: Hit Ratio, Latency, or Bandwidth? Have It All with Footprint 
Cache. In Proceedings of the 40th Annual International Symposium on Computer Architecture (ISCA '13). ACM, New York, NY, USA, 404-415. https://doi.org/10. $1145 / 2485922.2485957$

[54] Yoongu Kim, Vivek Seshadri, Donghyuk Lee, Jamie Liu, and Onur Mutlu. 2018 Exploiting the DRAM Microarchitecture to Increase Memory-Level Parallelism. arXiv preprint arXiv:1805.01966 (2018)

[55] N. Kirman, M. Kirman, R. K. Dokania, J. F. Martinez, A. B. Apsel, M. A. Watkins, and D. H. Albonesi. 2006. Leveraging Optical Technology in Future Bus-based Chip Multiprocessors. In 2006 39th Annual IEEE/ACM International Symposium on Microarchitecture (MICRO'06). 492-503. https://doi.org/10.1109/MICRO.2006. 28

[56] N. P. V. Krishna and P. Sen. 2017. Die level 3D heterogeneous integration of a microfluidic system. In 2017 IEEE 19th Electronics Packaging Technology Conference (EPTC). 1-3. https://doi.org/10.1109/EPTC.2017.8277519

[57] Ashok V Krishnamoorthy, Ron Ho, Xuezhe Zheng, Herb Schwetman, Jon Lexau, Pranay Koka, GuoLiang Li, Ivan Shubin, and John E Cunningham. 2009. Computer systems based on silicon photonic interconnects. Proc. IEEE 97, 7 (2009) 1337-1361.

[58] John H Lau. 2010. TSV manufacturing yield and hidden costs for 3D IC integration. In 2010 Proceedings 60th Electronic Components and Technology Conference (ECTC). IEEE, 1031-1042.

[59] Donghyuk Lee, Saugata Ghose, Gennady Pekhimenko, Samira Khan, and Onur Mutlu. 2016. Simultaneous multi-layer access: Improving 3D-stacked memory bandwidth at low cost. ACM Transactions on Architecture and Code Optimization (TACO) 12, 4 (2016), 63

[60] Donghyuk Lee, Samira Khan, Lavanya Subramanian, Saugata Ghose, Rachata Ausavarungnirun, Gennady Pekhimenko, Vivek Seshadri, and Onur Mutlu. 2017. Design-induced latency variation in modern DRAM chips: Characterization, analysis, and latency reduction mechanisms. Proceedings of the ACM on $\mathrm{Mea}$ surement and Analysis of Computing Systems 1, 1 (2017), 26.

[61] Donghyuk Lee, Yoongu Kim, Gennady Pekhimenko, Samira Khan, Vivek Se shadri, Kevin Chang, and Onur Mutlu. 2015. Adaptive-latency DRAM: Optimizing DRAM timing for the common-case. In 2015 IEEE 21st International Symposium on High Performance Computer Architecture (HPCA). IEEE, 489-501.

[62] Donghyuk Lee, Yoongu Kim, Vivek Seshadri, Jamie Liu, Lavanya Subramanian, and Onur Mutlu. 2013. Tiered-latency DRAM: A low latency and low cost DRAM architecture. In 2013 IEEE 19th International Symposium on High Performance Computer Architecture (HPCA). IEEE, 615-626.

[63] Donghyuk Lee, Lavanya Subramanian, Rachata Ausavarungnirun, Jongmoo Choi, and Onur Mutlu. 2015. Decoupled direct memory access: Isolating CPU and IO traffic by leveraging a dual-data-port DRAM. In 2015 International Conference on Parallel Architecture and Compilation (PACT). IEEE, 174-187.

[64] J. Lee, M. Zhu, K. Choi, J. H. Ahn, and R. Sharma. 2011. 3D network-on-chip with wireless links through inductive coupling. In 2011 International SoC Design Conference. 353-356. https://doi.org/10.1109/ISOCC.2011.6138783

[65] Jong Chern Lee, Jihwan Kim, Kyung Whan Kim, Young Jun Ku, Dae Suk Kim, Chunseok Jeong, Tae Sik Yun, Hongjung Kim, Ho Sung Cho, Yeon Ok Kim, et al. 2016. 18.3 A 1.2 V 64Gb 8-channel 256GB/s HBM DRAM with peripheral-basedie architecture and small-swing technique on heavy load interface. In 2016 IEEE International Solid-State Circuits Conference (ISSCC). IEEE, 318-319.

[66] Yongjun Lee, Jongwon Kim, Hakbeom Jang, Hyunggyun Yang, Jangwoo Kim, Jinkyu Jeong, and Jae W Lee. 2015. A fully associative, tagless DRAM cache. In ACM SIGARCH Computer Architecture News, Vol. 43. ACM, 211-222.

[67] Hao Li et al. 2015. A $25 \mathrm{~Gb} / \mathrm{s}, 4.4 \mathrm{~V}$-swing, AC-coupled ring modulator-based WDM transmitter with wavelength stabilization in $65 \mathrm{~nm}$ CMOS. IEEE fournal of Solid-State Circuits 50, 12 (2015), 3145-3159.

[68] Li Li, Pierre Chia, Paul Ton, Mohan Nagar, Sada Patil, Jie Xue, Javier Delacruz, Marius Voicu, Jack Hellings, Bill Isaacson, et al. 2016. 3D SiP with organic interposer for ASIC and memory integration. In 2016 IEEE 66th Electronic Components and Technology Conference (ECTC). IEEE, 1445-1450.

[69] Sheng Li, Jung Ho Ahn, Richard D Strong, Jay B Brockman, Dean M Tullsen, and Norman P Jouppi. 2009. McPAT: an integrated power, area, and timing modeling framework for multicore and manycore architectures. In Proceedings of the 42nd Annual IEEE/ACM International Symposium on Microarchitecture. ACM, 469-480.

[70] Teck Heng Lim, Takefumi Yoshikawa, Tony Tae-Hyoung Kim, et al. 2016. 2.31$\mathrm{Gb} / \mathrm{s} / \mathrm{ch}$ area-efficient crosstalk canceled hybrid capacitive coupling interconnect for 3-D integration. IEEE Transactions on Very Large Scale Integration (VLSI) Systems 24, 8 (2016), 2703-2711.

[71] Gabriel H Loh. 2008. 3D-stacked memory architectures for multi-core processors. In ACM SIGARCH computer architecture news, Vol. 36. IEEE Computer Society, 453-464.

[72] Gabriel H Loh. 2009. Extending the effectiveness of 3D-stacked DRAM caches with an adaptive multi-queue policy. In Proceedings of the 42nd Annual IEEE/ACM International Symposium on Microarchitecture. ACM, 201-212.

[73] Gabriel H Loh and Yuan Xie. 2010. 3D stacked microprocessor: Are we there yet? IEEE Micro 30, 3 (2010), 60-64.
[74] Samantha Donovan Luke England. June 19, 2018. RAdvanced Si Packaging Update.

[75] Tohoku-Microtec Makoto Motoyoshi. 2017. Status and roadmap of hybridization technologies relevant to future pixel detectors. SOIPIX2017 (2017).

[76] Milo MK Martin, Daniel J Sorin, Bradford M Beckmann, Michael R Marty, Min Xu, Alaa R Alameldeen, Kevin E Moore, Mark D Hill, and David A Wood. 2005. Multifacet's general execution-driven multiprocessor simulator (GEMS) toolset. ACM SIGARCH Computer Architecture News 33, 4 (2005), 92-99.

[77] High-Bandwidth Memory. 2015. Reinventing Memory Technology.

[78] Rick Merritt. 2017. Monolithic 3D Shows Promise, Challenges. https://www. eetimes.com/document.asp?doc id=1332490\#. [Online; accessed 03-01-2019].

[79] Micron. 2017. Hybrid Memory Cube. https://www.micron.com/products/hybridmemory-cube. [Online; accessed 03-01-2019].

[80] Kazuyuki Mitsukura, Tatsuya Makino, Keiichi Hatakeyama, Kenneth June Rebibis, Teng Wang, Giovanni Capuz, Fabrice Duval, Mikael Detalle, Andy Miller, and Eric Beyne. 2016. Packaging Material Evaluation for 2.5 D/3D TSV Application. Transactions of The fapan Institute of Electronics Packaging 9 (2016), E16-011.

[81] Sparsh Mittal and Jeffrey S Vetter. 2016. A survey of techniques for architecting DRAM caches. IEEE Transactions on Parallel and Distributed Systems 27, 6 (2016), $1852-1863$.

[82] Noriyuki Miura, Yusuke Koizumi, Yasuhiro Take, Hiroki Matsutani, Tadahiro Kuroda, Hideharu Amano, Ryuichi Sakamoto, Mitaro Namiki, Kimiyoshi Usami, Masaaki Kondo, et al. 2013. A scalable 3D heterogeneous multicore with an inductive ThruChip interface. IEEE Micro 33, 6 (2013), 6-15.

[83] Noriyuki Miura and Tadahiro Kuroda. 2007. A 1Tb/s 3W inductive-coupling transceiver chip. In 2007 Asia and South Pacific Design Automation Conference. IEEE, 92-93.

[84] Noriyuki Miura, Daisuke Mizoguchi, Mari Inoue, Takayasu Sakurai, and Tadahiro Kuroda. 2006. A 195-Gb/s 1.2-W inductive inter-chip wireless superconnect with transmit power control scheme for 3-D-stacked system in a package. IEEE fournal of Solid-State Circuits 41, 1 (2006), 23-34.

[85] Noriyuki Miura, Mitsuko Saito, and Tadahiro Kuroda. 2012. A 1 TB/s 1 pJ/b 6.4 QDR Inductive-Coupling Interface Between 65-nm CMOS Logic and Emulated 100-nm DRAM. IEEE fournal on Emerging and Selected Topics in Circuits and Systems 2, 2 (2012), 249-256.

[86] R. Morris, A. K. Kodi, and A. Louri. 2012. 3D-NoC: Reconfigurable 3D photonic on-chip interconnect for multicores. In 2012 IEEE 30th International Conference on Computer Design (ICCD). 413-418. https://doi.org/10.1109/ICCD.2012.6378672

[87] Naveen Muralimanohar, Rajeev Balasubramonian, and Norman P Jouppi. 2009. CACTI 6.0: A tool to model large caches. HP laboratories (2009), 22-31.

[88] A. Nowatzyk, Fong Pong, and A. Saulsbury. 1996. Missing the Memory Wall: The Case for Processor/Memory Integration. In 23rd Annual International Symposium on Computer Architecture (ISCA'96). 90-90. https://doi.org/10.1109/ISCA.1996. 10008

[89] T. Li N. Calabretta G. Guelbenzu de Villota R. Stabile O. Raz, C. Li. 2018. Scaling optical interconnects to meet the bandwidth density crunch. https://doi.org/10. $1117 / 12.2295759$

[90] Mike O'Connor, Niladrish Chatterjee, Donghyuk Lee, John Wilson, Aditya Agrawal, Stephen W Keckler, and William J Dally. 2017. Fine-grained DRAM: energy-efficient DRAM for extreme bandwidth systems. In Proceedings of the 50th Annual IEEE/ACM International Symposium on Microarchitecture. ACM, $41-54$.

[91] Takayuki Ohba. 2013. Wafer level three-dimensional integration (3DI) using bumpless TSV interconnects for tera-scale generation. In 2013 International Semiconductor Conference Dresden-Grenoble (ISCDG). IEEE, 1-4.

[92] Shreepad Panth, Sandeep Samal, Yun Seop Yu, and Sung Kyu Lim. 2014. Design challenges and solutions for ultra-high-density monolithic 3D ICs. In 2014 SOI3D-Subthreshold Microelectronics Technology Unified Conference (S3S). IEEE, 1-2.

[93] Ioannis A Papistas. 2018. Design Methodologies for Heterogeneous 3-D Integrated Systems. Ph.D. Dissertation. The University of Manchester (United Kingdom).

[94] Ioannis A Papistas and Vasilis F Pavlidis. 2015. Bandwidth-to-area comparison of through silicon vias and inductive links for 3-D ICs. In 2015 European Conference on Circuit Theory and Design (ECCTD). IEEE, 1-4.

[95] Mahavir S Parekh, Paragkumar A Thadesar, and Muhannad S Bakir. 2011. Electrical, optical and fluidic through-silicon vias for silicon interposer applications. In 2011 IEEE 61st Electronic Components and Technology Conference (ECTC). IEEE, 1992-1998.

[96] Vasilis F Pavlidis, Ioannis Savidis, and Eby G Friedman. 2017. Three-dimensional integrated circuit design. Newnes.

[97] D. Qiu, L. Cao, X. Wu, F. g. Hou, J. Zhang, and Q. Wang. 2015. Thermal management of 3D stacked dies with air convection and water cooling methods. In 2015 IEEE 65th Electronic Components and Technology Conference (ECTC). 839-844. https://doi.org/10.1109/ECTC.2015.7159690

[98] L. Ramini, D. Bertozzi, and L. P. Carloni. 2012. Engineering a BandwidthScalable Optical Layer for a 3D Multi-core Processor with Awareness of Layout Constraints. In 2012 IEEE/ACM Sixth International Symposium on Networks-onChip. 185-192. https://doi.org/10.1109/NOCS.2012.29 
[99] Mostafa Said, Mohamed El-Sayed, Farhad Mehdipour, and Nobuaki Miyakawa 2014. Keep-Out-Zone analysis for three-dimensional ICs. In Technical Papers of 2014 International Symposium on VLSI Design, Automation and Test. IEEE, 1-4.

[100] Sandeep Kumar Samal, Deepak Nayak, Motoi Ichihashi, Srinivasa Banna, an Sung Kyu Lim. 2016. Monolithic 3D IC vs. TSV-based 3D IC in 14nm FinFET technology. In 2016 IEEE SOI-3D-Subthreshold Microelectronics Technology Unified Conference (S3S). IEEE, 1-2.

[101] Stephan Schröder, Andreas C Fischer, Göran Stemme, and Frank Niklaus. 2013. Very high aspect ratio through silicon vias (TSVs) using wire bonding. In 2013 Transducers \& Eurosensors XXVII: The 17th International Conference on Solid-State Sensors, Actuators and Microsystems (TRANSDUCERS \& EUROSENSORS XXVII). IEEE, 167-170.

[102] O Seongil, Young Hoon Son, Nam Sung Kim, and Jung Ho Ahn. 2014. Row-buffer decoupling: A case for low-latency DRAM microarchitecture. In 2014 ACM/IEEE 41st International Symposium on Computer Architecture (ISCA). IEEE, 337-348.

[103] Amna Shahab, Mingcan Zhu, Artemiy Margaritov, and Boris Grot. 2018. Farewell My Shared LLC! A Case for Private Die-Stacked DRAM Caches for Servers. In 2018 51st Annual IEEE/ACM International Symposium on Microarchitecture (MICRO). IEEE, 559-572.

[104] Jiajun Shi, Deepak Nayak, Motoi Ichihashi, Srinivasa Banna, and Csaba Andras Moritz. 2016. On the Design of Ultra-High Density 14nm Finfet Based Transistor Level Monolithic 3D ICs. In 2016 IEEE Computer Society Annual Symposium on VLSI (ISVLSI). IEEE, 449-454.

[105] Anton Shilov. 2018. AMD Previews Epyc 'Rome' Processor: Up to 64 Zen 2 Cores. https://www.anandtech.com/show/13561/amd-previews-epyc-romeprocessor-up-to-64-zen-2-cores. [Online; accessed 11-14-2018].

[106] Dong J Shin, Kwan S Cho, Ho C Ji, Beom S Lee, Sung G Kim, Jin K Bok, Sang H Choi, Yong H Shin, Jung H Kim, Shin Y Lee, et al. 2013. Integration of silicon photonics into DRAM process. In Optical Fiber Communication Conference (OFC/NFOEC). IEEE, 1-3.

[107] Young Hoon Son, O Seongil, Yuhwan Ro, Jae W Lee, and Jung Ho Ahn. 2013. Reducing memory access latency with asymmetric DRAM bank organizations. In ACM SIGARCH Computer Architecture News, Vol. 41. ACM, 380-391.

[108] S Spiesshoefer, Z Rahman, G Vangara, S Polamreddy, S Burkett, and L Schaper 2005. Process integration for through-silicon vias. Fournal of Vacuum Science \& Technology A: Vacuum, Surfaces, and Films 23, 4 (2005), 824-829.

[109] Chen Sun, Chia-Hsin Owen Chen, George Kurian, Lan Wei, Jason Miller, Anant Agarwal, Li-Shiuan Peh, and Vladimir Stojanovic. 2012. DSENT-a tool connecting emerging photonics with electronics for opto-electronic networks-on-chip modeling. In 2012 IEEE/ACM Sixth International Symposium on Networks-on-Chip. IEEE, 201-210.

[110] Ishan G Thakkar and Sudeep Pasricha. 2014. 3D-Wiz: A novel high bandwidth, optically interfaced 3D DRAM architecture with reduced random access time. In 2014 IEEE 32nd International Conference on Computer Design (ICCD). IEEE, $1-7$.

[111] Martin Brox Wolfgang Spirkl Thomas Hein Dave Ovard Roy Greeff Dan Lin Michael Richter Peter Mayer Walt Moden Maksim Kuzmenka Mani Balakrishnan Milena Ivanov Manfred Plan Marcos Alvarez Gonzalez Bryce Gardiner Dong Soon Lim Tim Hollis, Salman Jiva and JÃürg Weller. 2018. 16Gb/s and Beyond with Single-Ended I/O in High-Performance Graphics Memorys. In DesignCon. Micron.

[112] Stefaan Van Huylenbroeck, Michele Stucchi, Yunlong Li, John Slabbekoorn, Nina Tutunjyan, Stefano Sardo, Nicolas Jourdan, Lieve Bogaerts, Filip Beirnaert, Gerald Beyer, et al. 2016. Small pitch, high aspect ratio via-last TSV module. In 2016 IEEE 66th Electronic Components and Technology Conference (ECTC). IEEE, 43-49.

[113] Wolfgang A Vitale, Montserrat Fernández-Bolaños, Armin Klumpp, Josef Weber, Peter Ramm, and Adrian M Ionescu. 2015. Ultra fine-pitch TSV technology for ultra-dense high-Q RF inductors. In 2015 Symposium on VLSI Technology (VLSI Technology). IEEE, T52-T53.

[114] Fengjuan Wang, Zhangming Zhu, Yintang Yang, Xiangkun Yin, Xiaoxian Liu, and Ruixue Ding. 2014. An effective approach of reducing the keep-out-zone induced by coaxial through-silicon-via. IEEE Transactions on Electron Devices 61, 8 (2014), 2928-2934.

[115] Zhehui Wang, Zhengbin Pang, Peng Yang, Jiang Xu, Xuanqi Chen, Rafael KV Maeda, Zhifei Wang, Luan HK Duong, Haoran Li, and Zhe Wang. 2017. MOCA An inter/intra-chip optical network for memory. In Proceedings of the 54th Annual Design Automation Conference (DAC) 2017. IEEE, 1-6.

[116] Sebastian Werner, Pouya Fotouhi, Roberto Proietti, and SJ Yoo. 2018. AWGR based optical processor-to-memory communication for low-latency, low-energy vault accesses. In Proceedings of the International Symposium on Memory Systems. ACM, 269-278.

[117] Sebastian Werner, Javier Navaridas, and Mikel Luján. 2017. A Survey on Optical Network-on-Chip Architectures. ACM Comput. Surv. 50, 6, Article 89 (Dec. 2017), 37 pages. https://doi.org/10.1145/3131346

[118] Ling Xie, Sunil Wickramanayaka, Ser Choong Chong, Vasarla Nagendra Sekhar, Daniel Ismeal, and Yong Liang Ye. 2016. 6um Pitch High Density Cu-Cu Bonding for 3D IC Stacking. In 2016 IEEE 66th Electronic Components and Technology Conference (ECTC). IEEE, 2126-2133.

[119] Yuan Xie and Jishen Zhao. 2015. Die-stacking architecture. Synthesis Lectures on Computer Architecture 10, 2 (2015), 1-127.

[120] Chen Yan and Emre Salman. 2018. Mono3D: Open source cell library for monolithic 3-D integrated circuits. IEEE Transactions on Circuits and Systems I: Regular Papers 65, 3 (2018), 1075-1085.

[121] K. Yao, Y. Ye, S. Pasricha, and J. Xu. 2017. Thermal-sensitive design and power optimization for a 3D torus-based optical NoC. In 2017 IEEE/ACM International Conference on Computer-Aided Design (ICCAD). 827-834. https://doi.org/10. 1109/ICCAD.2017.8203863

[122] CL Yu, CH Chang, HY Wang, JH Chang, LH Huang, CW Kuo, SP Tai, SY Hou, WL Lin, EB Liao, et al. 2011. TSV process optimization for reduced device impact on $28 \mathrm{~nm}$ CMOS. In 2011 Symposium on VLSI Technology-Digest of Technical Papers. IEEE, 138-139.

[123] Kunzhi Yu et al. 2016. A $25 \mathrm{~Gb} / \mathrm{s}$ hybrid-integrated silicon photonic sourcesynchronous receiver with microring Wavelength Stabilization. IEEE fournal of Solid-State Circuits 51, 9 (2016), 2129-2141.

[124] Xusheng Zhan, Yungang Bao, Christian Bienia, and Kai Li. 2017. PARSEC3. 0: A multicore benchmark suite with network stacks and SPLASH-2X. ACM SIGARCH Computer Architecture News 44, 5 (2017), 1-16.

[125] G. O Zhang. F van Roosmalen, and M. Graef. 2005. The paradigm of "more than Moore". In 2005 6th International Conference on Electronic Packaging Technology. 17-24. https://doi.org/10.1109/ICEPT.2005.1564646

[126] Tao Zhang, Cong Xu, Ke Chen, Guangyu Sun, and Yuan Xie. 2014. 3D-SWIFT: A high-performance 3D-stacked wide IO DRAM. In Proceedings of the 24th edition of the great lakes symposium on VLSI. ACM, 51-56.

[127] Y. Zhang, A. Dembla, Y. Joshi, and M. S. Bakir. 2012. 3D stacked microfluidic cooling for high-performance 3D ICs. In 2012 IEEE 62nd Electronic Component and Technology Conference. 1644-1650. https://doi.org/10.1109/ECTC.2012. 6249058

[128] Yu Zhang, Yi-Chun Ling, Yichi Zhang, Kuanping Shang, and SJ Ben Yoo. 2018. High-density wafer-scale 3-D silicon-photonic integrated circuits. IEEE fournal of Selected Topics in Quantum Electronics 24, 6 (2018), 1-10.

[129] Curtis Zwenger, Ron Huemoeller, JinHan Kim, DongJean Kim, WonChul Do, and SeongMin Seo. 2015. Silicon wafer integrated fan-out technology. Additional Papers and Presentations 2015, DPC (2015), 000217-000247. 